\title{
INITIATION AND EMERGING COMPLEXITY OF THE COLLAGEN NETWORK DURING PRENATAL SKELETAL DEVELOPMENT
}

\author{
S. Ahmed* and N.C. Nowlan \\ Department of Bioengineering, Imperial College London, London, United Kingdom
}

\begin{abstract}
The establishment of a complex collagen network is critical for the architecture and mechanical properties of cartilage and bone. However, when and how the key collagens in cartilage and bone develop has not been characterised in detail. The study provides a detailed qualitative characterisation of the spatial localisations of collagens I-III, V-VI and IX-XI in the mouse and their regional architecture variation over three developmentally significant time points: when the rudiment starts to form at E13.5 [Theiler stage (TS) 22], when mineralisation is present at E16.5 (TS25) and during the latest prenatal stage at E18.5 (TS27). Dynamic changes in collagen distribution between stages with the progression of the growth plate and mineralisation (particularly collagens I, II, V, X and XI) and dramatic changes in collagen structural organisation and complexity with maturation, especially for collagens II and XI, were observed. The future articular cartilage region was demarcated by pronounced collagens II and VI expression at TS27 and the emergence of collagens I, III, V, IX and XI in the tendon and its insertion site was observed. The present study revealed, for the first time, the emergence and maturation of key cartilage and bone collagens, in high resolution, at multiple locations across the entire rudiment, including the joint regions, at three of the most developmentally significant stages of skeletogenesis, furthering the understanding of disease and regeneration of skeletal tissues.
\end{abstract}

Keywords: Collagen I, collagen II, collagen III, collagen V, collagen VI, collagen IX, collagen X, collagen XI, humerus, skeletogenesis.

*Address for correspondence Dr Saima Ahmed, Department of Bioengineering, Imperial College London, London, SW72AZ, UK.

Email: ahmedsaima.sarkar@gmail.com

Copyright policy: This article is distributed in accordance with Creative Commons Attribution Licence (http://creativecommons.org/licenses/by-sa/4.0/).

\section{Introduction}

The extracellular matrix (ECM) provides tissues with their specific biochemical and mechanical properties. Resident cells synthesise and maintain the ECM and the ECM, in turn, regulates the cells' functions (Gelse et al., 2003). Cell-matrix interactions through specific receptor-ligand binding play critical roles in cell signalling, defining tissue boundaries and regulating both cell and tissue morphogenesis (Rozario and DeSimone, 2010). The cartilage ECM is composed of two main components that define its biochemical and mechano-physical properties: a collagen network, responsible for tensile strength, and proteoglycans, responsible for osmotic swelling and elastic properties (Gentili and Cancedda, 2009). The ECM is a key regulator of bone mechanical properties. Bone ECM is composed largely of calcium phosphate in the form of hydroxyapatite, with extensive collagen-I-rich organic matrix (Alford et al., 2015). Collagen fibrils appear first in the ECM of rudimentary cartilage, bone, tendons and ligaments soon after mesenchymal condensation (Kadler et al., 2008). During foetal and postnatal development, the fibrils change in dimensions and increase in abundance until they become the most abundant structural component of the adult skeletal tissues. As the tissues mature, the molecular organisation and the width as well as the orientation of the collagen fibrils change depending on the external forces experienced during load bearing (Blaschke et al., 2000).

Collagen II is the main matrix component in cartilage, but the minor collagens III, VI, IX, X and XI also contribute to the mature matrix (Eyre, 2002). Bone collagens mainly consist of collagens I and V (Niyibizi and Eyre, 1989). Abnormal amounts of all these collagens have been shown to have effects on cartilage or bone development (Rozario and DeSimone, 2010). For example, in humans, a mutation in the collagen I gene (which encodes the chains of type I procollagen) leads to osteogenesis imperfecta (von Der Mark, 2006), with a reduction 
in bone mass as well as an increase in bone fragility and multiple fractures (Forlino et al., 2011). Studies on Col2a1 null mice have shown that collagen II is required for the proper formation of articular cartilage, epiphyseal growth plate, endochondral bone and intervertebral discs (Aszodi et al., 1998; Li et al., 1995a). Mice with a mutation in Col2a1 develop a phenotype resembling human chondrodysplasias and tend to develop osteoarthritis (OA) in old age (Garofalo et al., 1991; Vandenberg et al., 1991). An OA-like phenotype can also be induced in mice by upregulating Col2a1 activity, with disruption of the collagen II fibril assembly (Garofalo et al., 1993). Moreover, targeted inactivation of Col2a1 prevents endochondral bone formation in mice (Li et al., 1995a). In humans, mutations of COL2A1 can contribute to osteochondrodysplasias and/or OA (Helminen et al., 2002). Disruptions in Col11a1 and Col11a2 have very different effects on murine susceptibility to OA. Premature termination of $\alpha 1(\mathrm{XI})$-chain mRNA translation in heterozygous cho/+ mice leads to OA-like changes (Olsen, 1995) whereas mice with targeted disruption of Col11a2 do not show any OA phenotype. However, in these latter mice, changes in growth plate morphology can be observed histologically (Li et al., 1995b). In mice, a deficiency in the major collagen $\mathrm{V}$ chain is fatal in early embryogenesis and is linked to an absence of collagen fibrils (Wenstrup et al., 2004). The number of collagen fibrils and collagen fibril diameter have been shown to be directly related to the collagen $\mathrm{V}$ gene dose (Beighton et al., 1998; Birk, 2001; Glimcher et al., 1980; Niyibizi and Eyre, 1989). Mice lacking collagen VI have delayed secondary ossification and reduced bone mineral density (Alexopoulos et al., 2009; Christensen et al., 2012). Col9a1 knockout mice have disrupted growth plates (Blumbach et al., 2009) and mice lacking collagen $X$ have severe impairment of haematopoiesis, indicating that collagen $X$ contributes to the establishment of the haematopoietic niche at the osteochondral junction (Sweeney et al., 2010). Damaged collagen fibrils are the first sign of overt cartilage degeneration characteristic of OA (Billinghurst et al., 2001; Poole et al., 2002; Stoop et al., 1999). Focal deposition of collagen III in the territorial matrix is significantly increased in OA cartilage when compared to normal cartilage (Hosseininia et al., 2016) and collagen $X$ expression is elevated in human and mouse osteoarthritic cartilage, due to chondrocyte hypertrophy and cartilage calcification (Kamekura et al., 2005; Walker et al., 1995). Mice in which the function of collagens VI, IX or XI has been compromised show accelerated development of OA (Alexopoulos et al., 2009; Holyoak et al., 2018; Parsons et al., 2011).

A small number of studies have described collagen localisation patterns during skeletal rudiment development. Archer et al. (1994) described the spatiotemporal distribution of collagens I and II within the developing joint of the embryonic chick. Prior to cavitation, collagen I immunopositivity is mildly detectable in the joint interzone, which then becomes restricted to the developing joint capsule and articular surfaces after cavitation. In contrast, collagen II is present in the interzone prior to cavitation and its presence in the interzone diminishes with the progression of cavitation. Following complete cavitation, collagen II is restricted only to the epiphyseal cartilage. Morrison et al. (1996) described the tissue distribution patterns of collagens I, II, III, VI and X in the developing knee joint cartilage of a marsupial model system. Similar to Archer et al. (1994), they found that collagen I localisation in the epiphyseal cartilage weakens with development. Collagen II is found throughout the cartilage while collagen III is only present at the insertion sites of major ligaments and tendons and within the perichondrium/periosteum. Collagen VI is seen throughout the cartilage, restricted to the pericellular matrix. Collagen $X$ is confined to the hypertrophic chondrocytes and its expression precedes the start of endochondral bone formation (Morrison et al., 1996). A later study by Shobam et al. (2016) further described the collagen I deposition pattern with respect to vascular patterning and bone morphology. Collagen I deposition initiates with the formation of the bone collar and, as the bone collar expands radially, newly deposited collagen I is found in its outer layer. MüllerGlauser et al. (1986) showed that both collagen II and IX are found in the cartilaginous, but not in the mineralised, regions of 17 -d-old chick long bones. Studies of cultured chondrocytes in vitro have shown that chondrocytes of the developing human femoral head synthesise collagen III (Treilleux et al., 1992). Finally, Foolen et al. (2008) showed that collagen fibre orientation in the developing chick periosteum and perichondrium aligns preferentially in the direction of the tissue growth. These studies are currently the best data available on the emergence of collagens during skeletogenesis. However, they do not describe the structural organisation of all key cartilage and bone collagens across the various regions of the rudiment or across the entire range of the rudiment development. This was the gap addressed in the present study.

The present study provides a comprehensive description of how the structural organisations and tissue distributions of collagens type I, II, III, V, VI, IX, $X$ and XI emerge and change in the humerus during prenatal development of the mouse, at Theiler stages (TS) 22, TS25 and TS27 (typically embryonic days 13.5, 16.5 and 18.5, respectively). These eight collagens were chosen because cartilage and/or bone abnormalities result when their distribution and structure are altered in human pathologies or in animal models (Rozario and DeSimone, 2010). Understanding the emergence of composition and architecture of collagens during cartilage and bone development will advance the understanding of the development, maturation and degradation of cartilage and bone and open avenues towards novel regenerative medicine strategies for diseased cartilage 
and bone, through recapitulation of developmental processes.

\section{Materials and Methods}

\section{Tissue collection}

All experiments were performed in accordance with European legislation (Directive 2010/63/EU). Mouse embryos (C57BL/6 strain) were harvested and staged according to Theiler stages TS22, TS25 and TS27 (typically embryonic day 13.5, 16.5 and 18.5, respectively). Forelimbs were dissected and processed for cryosectioning. Each collagen type was characterised in three distinct embryos. In the results, one representative sample is shown for each collagen but the results described were consistent for all the three individual replicates studied.

\section{Histology and collagen detection by immunofluorescence}

Forelimbs were processed in a sucrose gradient (15\% and $30 \%$ sucrose) and embedded in a medium containing $50 \%$ sucrose and $50 \%$ optimal cutting temperature (OCT) embedding matrix (Agar Scientific, Stansted, UK). $12 \mu$ m-thick tissue sections were cut using a cryostat and fixed for $10 \mathrm{~min}$ at room temperature in $4 \%(\mathrm{w} / \mathrm{v})$ paraformaldehyde, while agitating. For histology, sections were stained with toluidine blue (Sigma-Aldrich) for $10 \mathrm{~s}$ and washed in water. Following air-drying, sections were imaged by transmitted illumination using a light microscope (Yenway EX30; Life Sciences Microscope, Glasgow, UK). Images were used to identify the regions of interest. For immunofluorescence, tissues were permeabilised with $0.1 \%$ Tween 20 (SigmaAldrich)/1 \% dimethyl suphoxide (DMSO; SigmaAldrich) in phosphate-buffered saline (PBS), blocked with $5 \%$ (v/v) normal goat serum (Sigma-Aldrich) and incubated overnight at $4{ }^{\circ} \mathrm{C}$ with a primary antibody against the specific collagen type (1: 50 dilution; antibody details provided in Table 1). The next day, tissues were washed and incubated for
$2 \mathrm{~h}$ at room temperature with secondary antibody ( $1: 200$ dilutions) (antibody details provided in Table 1) and 4',6-diamidino-2-phenylindole (DAPI, SigmaAldrich) (1:1,000 dilution). Rabbit anti-mouse (Alexa Fluor $^{\circledR} 488$ ) was used for collagen II and goat antirabbit $\left(\mathrm{Cy}^{\circledR}{ }^{\circledR}\right)$ was used for all other collagens. Initially, experiments for all collagens were performed without digesting the tissue sections with hyaluronidase (HA). As no signal was detected for collagens III, IX and XI, the experimental protocol was optimised for these collagens by digesting the tissue sections with $10 \mathrm{mg} / \mathrm{mL}$ bovine testicular HA (Sigma-Aldrich) for $1 \mathrm{~h}$ prior to incubation with the primary antibody. Following this digestion step, collagen IX expression was only detected in the perinuclear region. Then, another digestion protocol using $10 \mathrm{mg} / \mathrm{mL} \mathrm{HA}$ in sodium acetate buffer [ $10 \mathrm{mM}$ sodium acetate (SigmaAldrich), $10 \mathrm{mM}$ ethylenediaminetetraacetic acid (EDTA; Sigma-Aldrich), $\mathrm{pH}$ 6.0] for an alternative collagen IX antibody (see Table 1) was tested.

\section{Image acquisition by confocal laser scanning microscopy and analysis}

The humerus was the focus of the study, with five regions chosen for detailed inspection; namely, the humeral head, the proliferative and hypertrophic regions of the growth plate, the mineralised region and the humeral condyles (Fig. 1). Since no discernible growth plate region was present at TS22 (Fig. 1), only the humeral head, mid-diaphysis (primary ossification centre) and humeral condyles were assessed at this stage. Direct fluorescence acquisition of labelled tissue sections was performed using a Zeiss LSM 510 inverted confocal laser scanning microscope equipped with blue-diode $405 \mathrm{~nm}$, argon 458-514 nm, helium and neon $543 \mathrm{~nm}$ and heliumneon $633 \mathrm{~nm}$ lasers. An overall image of the entire humerus was acquired with a $\times 10$ objective (PlanNeofluar $10 \times / 0.30$ ) and higher magnification images of the specific regions were acquired using a $\times 63$ oil objective (Plan-Apochromat $63 \times / 1.4$ ). The argon and helium-neon laser multi-track protocol was used (Eltawil et al., 2018). This protocol allowed alternate

Table 1. List of antibodies used and their product details.

\begin{tabular}{|c|c|c|}
\hline Antibody & Supplier & Catalogue number \\
\hline Collagen I & Abcam & ab34710 \\
\hline Collagen II & Sigma-Aldrich & MAB8887 \\
\hline Collagen III & Abcam & ab7778 \\
\hline Collagen V & Abcam & ab7046 \\
\hline Collagen VI & Abcam & $\mathrm{ab} 6588$ \\
\hline Collagen IX & Abcam & ab134568 \\
\hline Collagen IX & Thermo Fisher Scientific & PA5-38886 \\
\hline Collagen $X$ & Abcam & $\mathrm{ab} 58632$ \\
\hline Collagen XI & Invitrogen & PA5-77258 \\
\hline Goat anti-rabbit $\left(\mathrm{Cy}^{\circledR}\right)$ & Abcam & ab6939 \\
\hline Rabbit anti-mouse (Alexa Fluor ${ }^{(488)}$ & Abcam & ab150125 \\
\hline
\end{tabular}




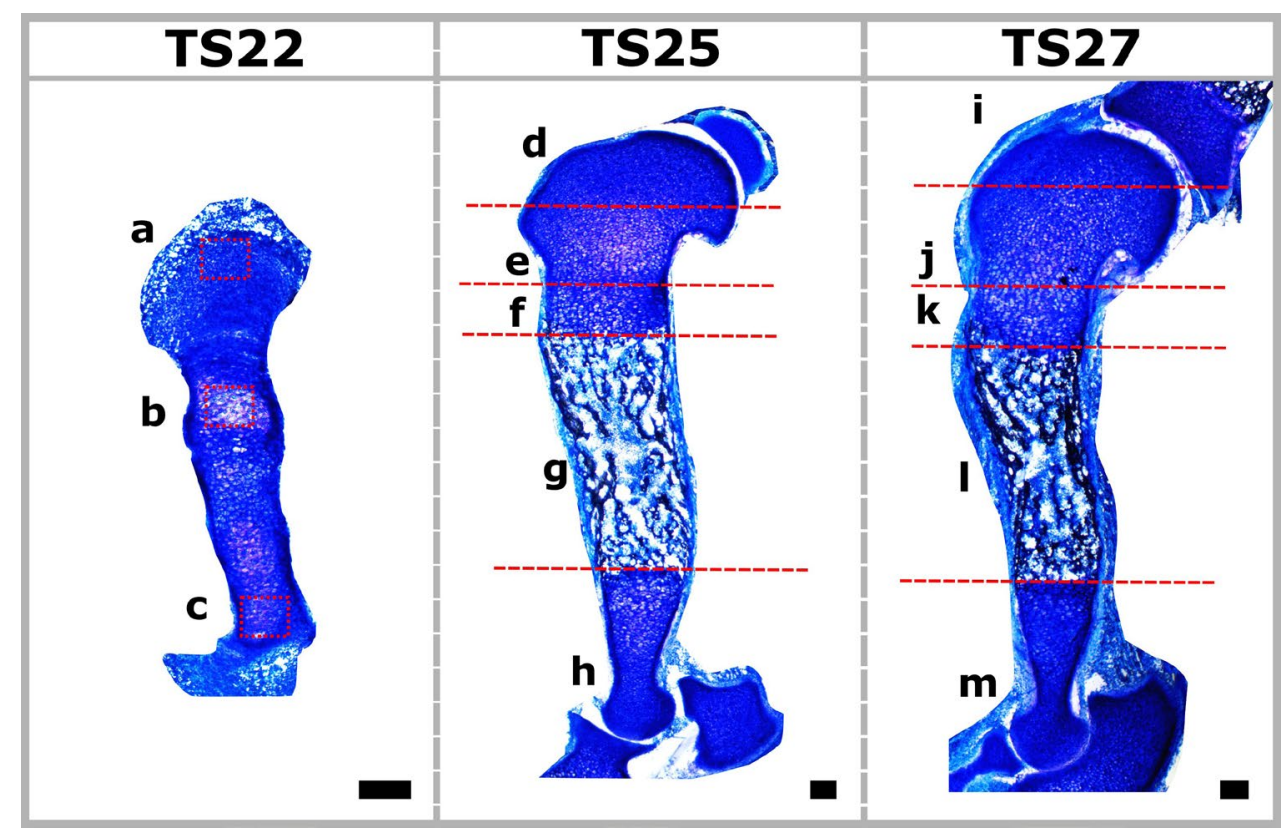

Fig. 1. Representative histological sections of the murine forelimb at TS22, TS25 and TS27. Sections were stained with toluidine blue. At TS22, the humeral head and the humeral condyles were still continuous, with the scapula and radius/ulna providing no evidence of cavitation. At this stage, three regions within the humerus were chosen for detailed assessment: (a) humeral head, (b) mid-diaphysis and (c) humeral condyles (red boxes). At TS25 and TS27, five regions within the humerus were chosen for detailed assessment: (d,i) humeral head, $(\mathbf{e}, \mathbf{j})$ proliferative and $(\mathbf{f}, \mathbf{k})$ hypertrophic regions of the growth plate, $(\mathbf{g}, \mathbf{l})$ mineralised region and $(\mathbf{h}, \mathbf{m})$ humeral condyles. Red dotted lines indicate the approximate boundaries between the regions examined. Scale bars: $100 \mu \mathrm{m}$.

excitation of DAPI $(\lambda \mathrm{ex}=358 \mathrm{~nm}, \lambda \mathrm{em}=461 \mathrm{~nm}$ and secondary antibody fluorophores such as Cy3 $(\lambda \mathrm{ex}=550 \mathrm{~nm}, \lambda \mathrm{em}=570 \mathrm{~nm})$ and, thereby, dual visualisation of the nuclei and collagen molecules within individual optical sections. The optimal gain of the fluorescence photomultiplier was manually adjusted before acquiring an image to avoid pixel saturation and obtain optimal imaging of each optical section. The diameter of the pinhole was set to 1 Airy unit (diameter which allowed rejection of out-of-focus light) for detection of both fluorophores. Using these parameters, the humerus of the prenatal forelimb was imaged in multiple optical sections. Where no obvious differences in collagen distribution were observed across the rudiment, only the humeral head was imaged at higher magnification. Within each region, confocal images were taken based on optimal visibility of prominent collagen architecture and in order to include perichondrial or periosteal immunolocalisation, where appropriate. Optical sections were reconstructed using Fiji (Image J) (Paletzki and Gerfen, 2015) to produce confocal projections. The "smooth" filter was applied to some of the fluorescent images in order to reduce the intensity variation between a pixel and the next one and reduce the noise in the images. This was done by replacing each pixel with the average of its immediate neighbours. Where it was not possible to capture the entire rudiment in one image using the $\times 10$ objective, individual images were taken, processed using Fiji (ImageJ) and stitched using Inkscape (Yuan et al., 2016).

\section{Results}

\section{Collagen I}

At TS22, collagen I was present at the proximal and distal ends of the rudiment with more pronounced expression at the proximal end (Fig. 2i). It was prominent in the perichondrium, with more marked expression proximally and at the mid-diaphyseal perichondrium (Fig. 2i). Perichondrial cells were embedded in the collagen I matrix (Fig. 2a). Mild immunopositivity was detected in some regions of the cartilage (Fig. 2i, filled arrows). By TS25, strong immunopositivity was detected in the mineralised regions (Fig. 2ii) with thick collagen I bundles (Fig. $2 b$ ). Staining intensity was diminished in the rest of the cartilage when compared to TS22 and mild immunopositivity was detected in the perichondrium (Fig. 2ii). At TS27, collagen I was detected throughout the mineralised region and was more pronounced in the perichondrium when compared to TS25 rudiments (Fig. 2iii). At this stage (TS27), collagen I bundles in the mineralised regions had two different organisations: (1) a lattice-like organisation towards the core (Fig. 2c) and (2) randomly oriented thicker bundles closer to the surface (Fig. 2d), with increased spacing between individual bundles when compared to TS25.

\section{Collagen II}

At TS22, collagen II was detected throughout the ECM of the developing rudiment, with milder immunolocalisation in the proximal and distal 
ends and mid-diaphysis (Fig. 3i). With maturation (TS25 and TS27), collagen II was retained only in the non-mineralised cartilage and perichondrium with a progressive reduction in collagen II expression nearest the advancing marrow cavity region of the growth plate (Fig. 3ii,iii). At higher magnification, striking differences in collagen II structure and organisation became apparent, both between regions and between stages. At TS22, collagen II staining was observed throughout the ECM (Fig. 3a) but the strongest staining was detected as either fine circular bands (Fig. 3a, hollow arrows) or close to the chondrocytes (Fig 3a, asterisks). In the TS22 mid-diaphysis (Fig. 3b) and humeral condyles (Fig. 3c), collagen II was present only at the edges of the nuclei with a stronger signal in the humeral condyles than in the mid-diaphysis. In the TS25 humeral head, the collagen II bundles started to form an oriented meshwork, where the chondrocytes were embedded in the mesh (Fig. 3d). A similar, although less pronounced, architecture was present in the proliferative region of the growth plate (Fig. 3e) and
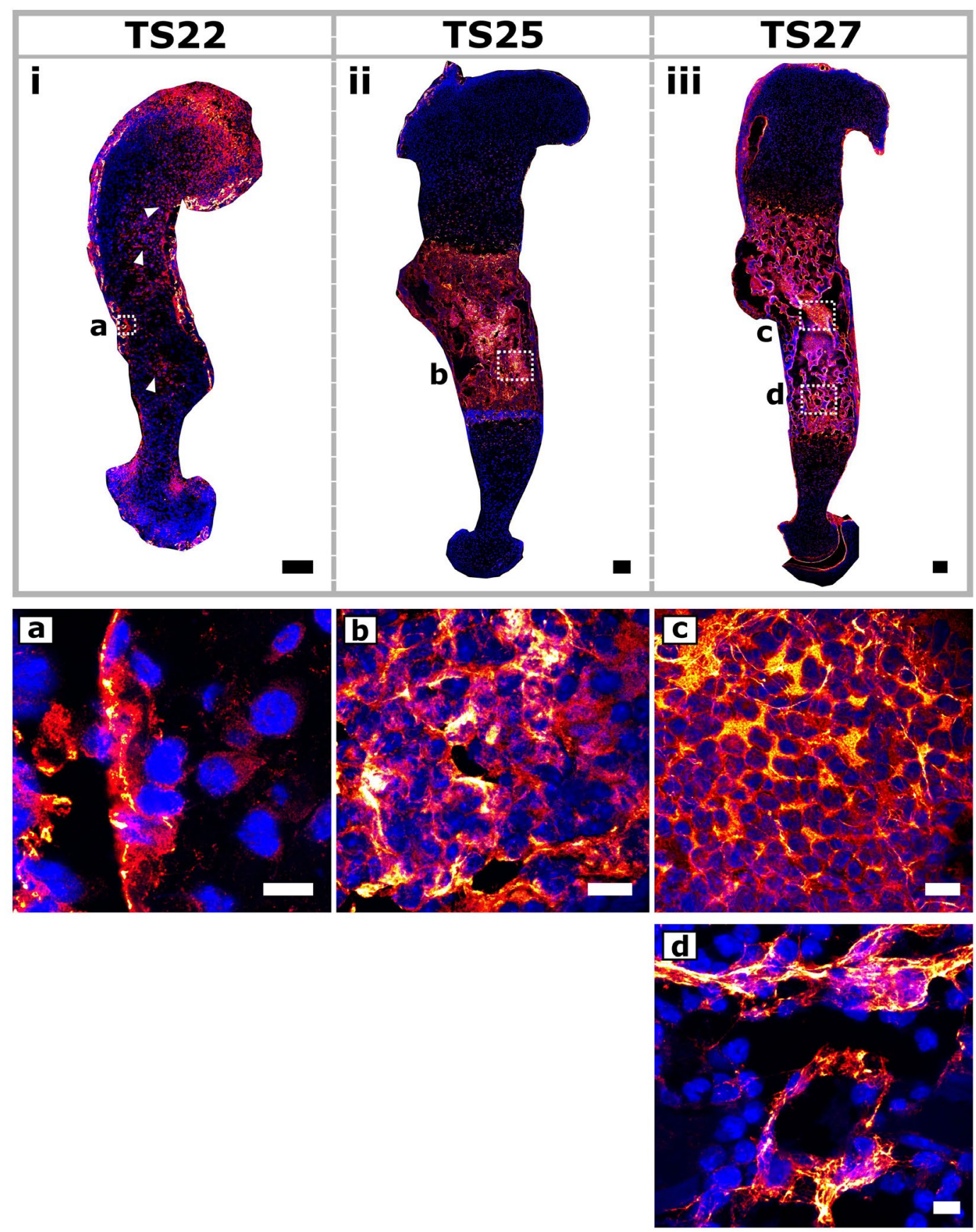

\section{DAPI Collagen I}

Fig. 2. Collagen I was primarily present in the perichondrium and the mineralising cartilage. (i) Filled arrows indicate mild immunopositivity for collagen I in the cartilage. (a) Perichondrial cells were embedded in the collagen I matrix at TS22. (b) Thick collagen I bundles were detected in the mineralised regions of the rudiments at TS25. (c,d) Two different collagen bundle organisations were observed at TS27. White dotted boxes represent the specific areas where the higher magnification images were taken. Scale bars: $100 \mu \mathrm{m}$ for i-iii and $10 \mu \mathrm{m}$ for a-d. 

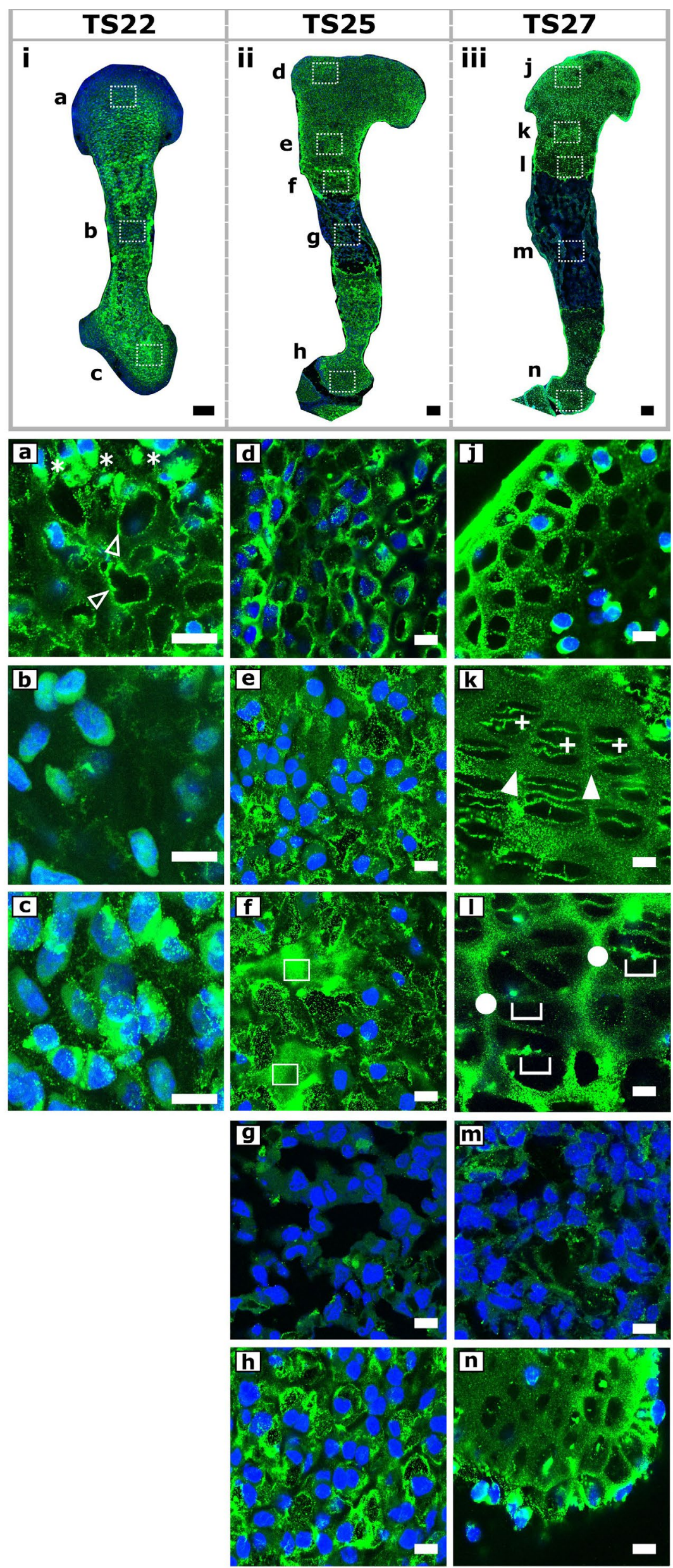

DAPI

\section{Collagen II}

Fig. 3. Collagen I I immunopositivity was detected throughout the rudiment except in the mineralising cartilage at all stages with a progressive reduction in collagen II expression near the advancing marrow cavity region of the growth plate. A fibrillar framework was present in the TS25 and TS27 (d,j) humeral head and $(\mathbf{h}, \mathbf{n})$ condyles. (k1) At TS27, collagen II matrix structural organisation was also well-defined in the growth plate regions. White dotted boxes in i-iii represent the specific areas where higher magnification images were taken. (a) Hollow arrows: fine circular bands of collagen II; asterisks: dense collagen II matrix distributed in the perinuclear regions. (f) Squares: dense fibres in the growth plate. (k) Filled arrows: longitudinal septa distribution; plus signs: transverse septa distribution. (1) Circles: thick bundles in the longitudinal septa of the hypertrophic region of growth plate; single-edged bars: expression in transverse septa of hypertrophic region of growth plate. Scale bars: $100 \mu \mathrm{m}$ for i-iii and $10 \mu \mathrm{m}$ for $\mathbf{a}-\mathbf{n}$. 
in the humeral condyle (Fig. 3h). Dense fibres (Fig. 3f, squares) were seen in the hypertrophic region of the growth plate. In the TS27 humeral head and condyles, the collagen II bundles were more prominent than in previous stages and highly organised forming a fibrillar network (Fig. 3j,n). In the proliferative region of the TS27 growth plate, collagen II was present both in the longitudinal [matrix between individual chondrocyte columns (Fig. 3k, filled arrows)] and transverse septa [matrix between the cells of a column (Fig. 3k, plus signs)]. In the hypertrophic region, the collagen II bundles were more pronounced in the longitudinal septa (Fig. 31, circles), compared to the transverse septa (Fig. 31, single-edged bars).

\section{Collagen III}

Collagen III was detected throughout the rudiment, including the perichondrium and periosteum, at all three stages (Fig. 4i-iii). At TS22, the strongest immunopositivity was observed at the proximal and distal ends of the rudiment but this did not persist across development. At TS22, collagen III was only present surrounding individual chondrocyte nuclei without any obvious difference in distribution pattern between the three regions that were assessed (Fig. 4a-c). At TS25 and TS27, collagen III was primarily present in the pericellular matrix with cristae-like structures (folding in the inner membrane) visible within each chondron (Fig. 4d-f,h-1, hollow arrows). At TS25, in the proliferative and hypertrophic regions of the growth plate, collagen III was present in the longitudinal septa (Fig. 4e,f, asterisks), while by TS27, this immunopositivity in the longitudinal septa was weakened (Fig. 41, filled arrow). Collagen III was randomly oriented and striated in the mineralised regions (Fig. 4g,m).

\section{Collagen V}

At TS22, collagen V was expressed throughout the diaphysis of the developing rudiment and was absent from the epiphysis and the perichondrium (Fig. 5i). At both TS25 and TS27, collagen V immunopositivity was detected in the mineralised region of the rudiment including the bone collar and was also present in the perichondrium (Fig. 5ii,iii). At TS22, collagen $\mathrm{V}$ appeared to be present in the pericellular matrix surrounding individual chondrocytes (Fig. 5a). At TS25, the collagen $\mathrm{V}$ pericellular matrix started to become connected to form a dense network (Fig. $5 b)$ and this fibrillar network of collagen $\mathrm{V}$ persisted at TS27, without any pronounced orientation (Fig. 5c).

\section{Collagen VI}

Collagen VI was detected throughout the rudiment and perichondrium at all three stages, with the strongest immunopositivity observed at the proximal and distal ends of the rudiment (Fig. 6i-iii). Among the three stages studied, proximal and distal end immunolocalisation was stronger at TS22 (Fig. 6i, black arrows). At TS22, collagen VI staining was detected in the ECM (Fig. 6a) but the strongest staining was observed in the pericellular matrix of the chondrocytes in all three regions (Fig. 6ac). In the TS25 humeral head, collagen VI was present in the pericellular matrix surrounding individual chondrocytes (Fig. 6d), with a dense, layered appearance within each chondron (Fig $6 \mathbf{d}$, hollow arrows). In the proliferative region of the growth plate, collagen VI was present in the pericellular matrix between chondrocyte columns, exhibiting a cristae-like structure (Fig 6e, asterisks). In the hypertrophic region, regions of collagen VI expression in the chondrons were enlarged and dense (Fig. 6f). In the TS25 mineralised region, collagen VI bundles were less pronounced, appeared fibrillar with a dominant orientation in the proximo-distal axis of the rudiment (Fig. 6g). In the humeral condyle, collagen VI formed a dense cylindrical ring around individual chondrocytes (Fig 6h, squares). At TS27, the collagen VI matrix of the humeral head was dense and compact surrounding individual chondrocytes and had cristae-like structures (Fig 6j, filled arrows). All the other regions had similar collagen VI distribution patterns to what was observed at TS25 (Fig. 6j-n).

\section{Collagen IX}

Collagen IX distribution was examined using three different protocols:

- addition of collagen IX primary antibody (ab134568) without HA digestion of tissue sections;

- addition of primary antibody (ab134568) following tissue digestion with HA (10 mg/mL);

- addition of primary antibody (PA5-38886) following tissue digestion with HA $(10 \mathrm{mg} / \mathrm{mL})$ in sodium acetate buffer.

No collagen IX expression was seen with the first protocol. Using the second protocol, collagen IX expression was observed throughout the rudiment, including the perichondrium, at all three stages (Fig. 7i-iii). Stronger immunopositivity was observed at the proximal and distal ends of the TS22 rudiment (Fig. 7i, black arrow), but this did not persist across development. At higher magnification, collagen IX was observed as a dense matrix around individual chondrocyte nuclei. There were no obvious differences in collagen IX matrix distribution at higher magnification either between the regions or the stages (Fig. 7a-c). When collagen IX expression was examined using the third protocol (PA5-38886), the same expression pattern as with ab134568 was observed (data not shown).

\section{Collagen $\mathrm{X}$}

Collagen $X$ immunopositivity was detected in the mid-diaphysis at TS22 (Fig. 8i) and in the growth plates at TS25 (Fig. 8ii) and TS27 (Fig. 8iii). At higher magnification, a hexagonal lattice-like framework was observed at TS22 (Fig. 8a). At TS25, collagen X matrix surrounded enlarged hypertrophic chondrocytes and was localised in the capsule or membrane-like 

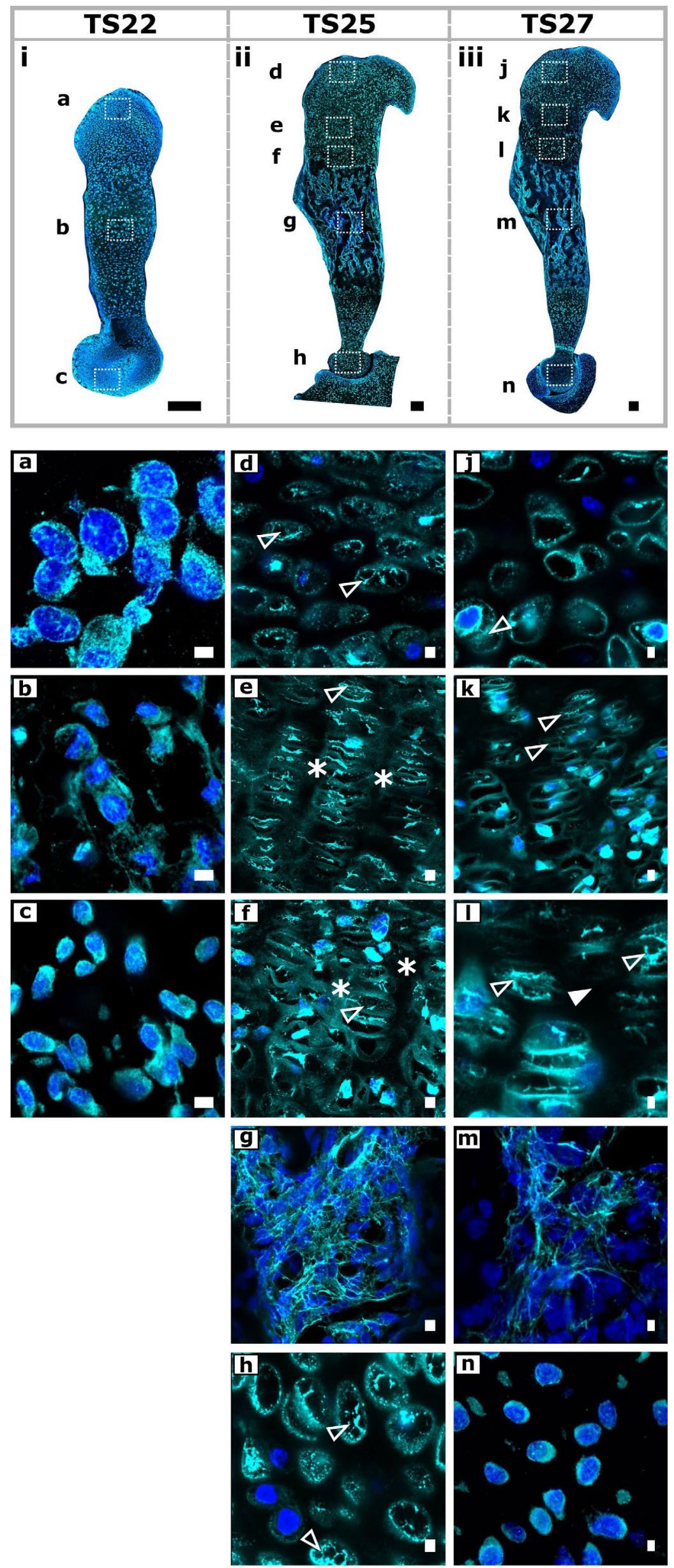

DAPI

Collagen III
Fig. 4. Collagen III was present throughout the rudiment and perichondrium at all three stages, localised primarily in the pericellular matrix, with distinct cristae-like structures within individual chondrons. White dotted boxes represent the specific areas where the higher magnification images were taken. (d-f; h-l) Hollow arrows: cristae-like structure (folding in the inner membrane) visible within each chondron. (e,f) Asterisks: distribution in the longitudinal septa. (1) Filled arrows: weakening of immunopositivity in the longitudinal septa. Scale bars: $100 \mu \mathrm{m}$ for i-iii and $5 \mu \mathrm{m}$ for a-n. 
configuration around each cell (asterisks, Fig. 8b). At TS27, collagen $X$ matrix had a columnar arrangement in the pre-hypertrophic zone (Fig 8c, upper half, hollow arrows) and a convex arrangement in the hypertrophic region of the growth plate (Fig 8c, lower half, filled arrows).

\section{Collagen XI}

Collagen XI was present throughout the rudiment at all three stages (Fig. 9i-iii), with less pronounced staining in the mineralising cartilage. At TS22, collagen XI bundles were organised in a fibrillar network and there was no difference in their organisation between the regions (Fig. 9a-c). This network organisation was retained in the humeral head and condyles at both TS25 (Fig. 9d,h) and TS27 (Fig. 9j,n). In the proliferative region of the TS25 growth plate, collagen XI was present in both the transverse (Fig 9e,10c,d, hollow arrows) and longitudinal (Fig. 9e,10c,d, filled arrows) septa. Each longitudinal septum contained long (Fig. 9e, 10c,d, filled arrows) and short (Fig. 9e, asterisks) collagen XI fibres, oriented parallel to each other, with small inter-fibre spaces. The characteristic shorter fibres were present in the TS25 hypertrophic region (Fig. 9f, asterisks) and in the TS27 growth plate regions (Fig. 9k,1,10e,f, asterisks). No obvious matrix organisation was present in the mineralised regions (Fig. 9g,m). At TS27, in the growth plate proliferative region, collagen XI organisation in both types of septa was more pronounced (Fig 9k, square, 10e,f filled and hollow arrows) when compared to TS25.

Collagen distribution at the developing humeroulnar joint

Collagen distribution was also assessed at the developing humero-ulnar joint (Fig. 11). Prior to cavitation (TS22), collagen I was present throughout the matrix of the epiphyseal cartilage (Fig. 11a, solid
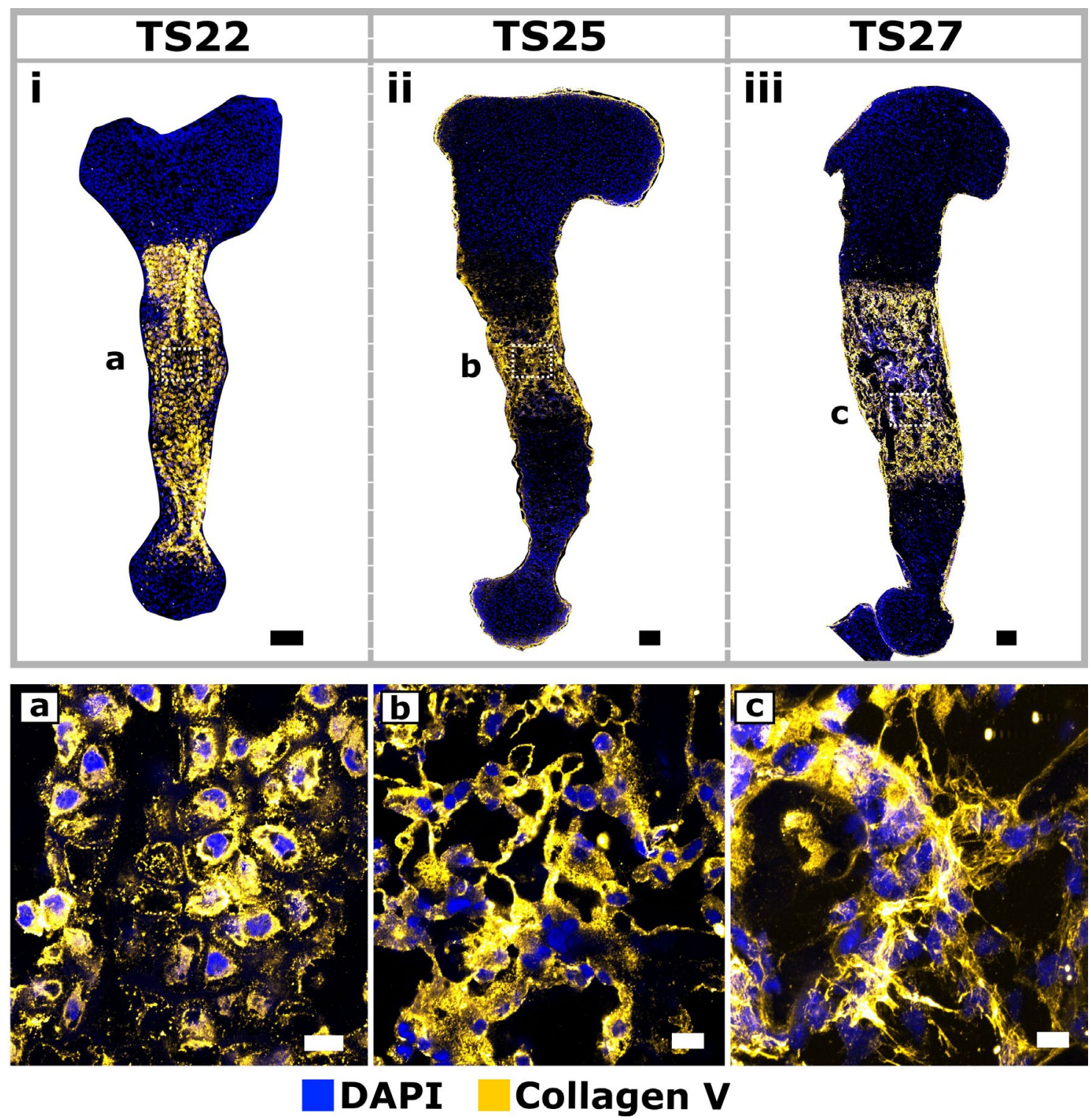

Fig. 5. Collagen V was present in the diaphysis at TS22 and in the perichondrium and mineralised regions at TS25 and TS27, with a fibrillar organisation in the mineralised regions. White dotted boxes represent the specific areas where the higher magnification images were taken. Scale bars: $100 \mu \mathrm{m}$ for i-iii and $10 \mu \mathrm{m}$ for a-c. 

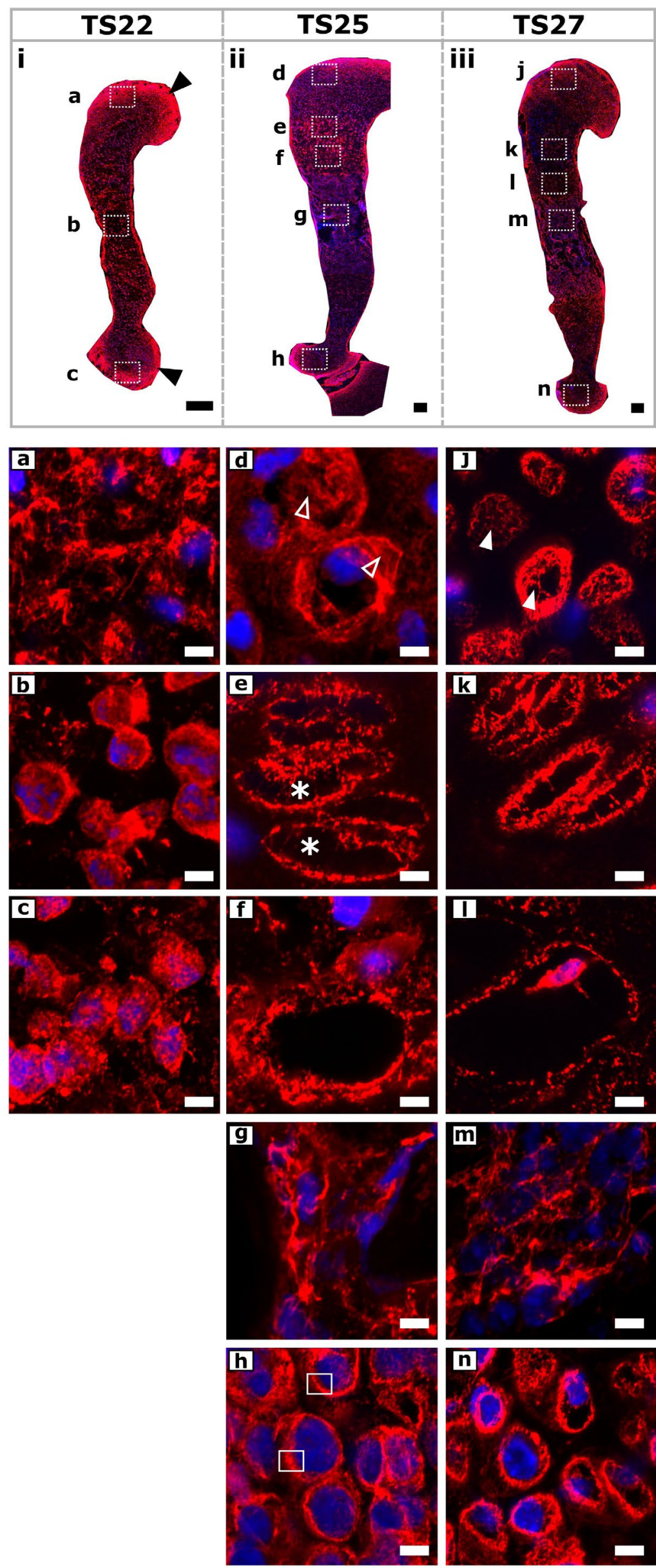

DAPI

Collagen VI
Fig. 6. Collagen VI was only present in the perichondrium and pericellular matrix of the foetal humerus at all three stages, localised primarily to the pericellular matrix. (a-c) Chondron initiation was observed at TS22. Full chondrons encapsulating individual chondrocytes were observed at (d-h) TS25 with increased chondron complexity at (j-n) TS27. White dotted boxes represent the specific areas where the higher magnification images were taken. (i) Filled black arrows: strong collagen VI immunopositivity in the proximal and distal ends of the TS22 rudiment. (d) Hollow arrows: dense layered appearance of collagen VI in the pericellular matrix of the humeral head. (e) Asterisks: individual chondrons exhibiting cristae-like structure. (h) Squares: dense cylindrical rings of collagen VI around individual chondrocytes. (j) Filled arrows: cristae-like structures in the TS27 chondrons. Scale bars: $100 \mu \mathrm{m}$ for $\mathbf{i}$-iii and $5 \mu \mathrm{m}$ for a-n. 
arrows) but became confined to the perichondrium post-cavitation [TS25 (Fig. 11b, solid arrows) and TS27 (Fig. 11c, solid arrows)]. At TS22 and TS25, collagen II was present throughout the epiphyseal cartilage but no signal for this collagen was detected at the future articular cartilage region (Fig. 11d,e, hollow arrows). At TS27, collagen II was expressed both in the future articular cartilage region and the epiphysis (Fig. 11f, solid arrows), clearly demarcating the future articular cartilage region. Collagens III (Fig. 11g-i, solid arrows), VI (Fig. 11m-o, solid arrows) and XI (Fig. 11v-x, solid arrows) were consistently expressed in both the future articular cartilage region and the epiphyseal cartilage. Collagen IX (Fig. 11pr, solid arrows) was consistently expressed in the epiphyseal cartilage. Collagen V was not expressed in the joint region at TS22 (Fig. 11j, hollow arrows) but was strongly expressed in the perichondrium at TS25 (Fig. 11k, solid arrows) and TS27 (Fig. 111, solid arrows), clearly demarcating the rudiment boundaries. Collagen $\mathrm{X}$ was not expressed in the joint region (Fig. 11s-u, hollow arrows).

\section{Collagen localisation in the tendon}

At TS22, collagens I (Fig. 11a, arrowhead) and VI (Fig. 11m, arrowhead) were strongly expressed in the tendon, while collagen III (Fig. 11g, arrowhead), IX (Fig. 11p, arrowhead) and XI (Fig. 11v, arrowhead) were most prominently expressed at the insertion site. These expression patterns persisted at TS25 (Fig. 11h,q,w, arrowhead) and TS27 (Fig. 11i,r, $\mathbf{x}$, arrowhead). Collagen $\mathrm{V}$ was not present in the tendon at TS22 (Fig. 11j), with expression increasing starting from TS25 (Fig. 11k, l, arrowhead).

\section{Discussion}

Assessing ECM composition and architecture using high-resolution confocal microscopy enabled elucidation of a highly resolved spatial distribution and regional variation in collagen architecture not previously reported for an entire rudiment. Previous immunofluorescence studies have localised collagens I, II, III, VI, IX and X in the embryonic limb (Archer et al., 1994; Castagnola et al., 1988; Duance et al., 1982; Evans et al., 1983; Foolen et al., 2008; Hartmann et al., 1983; Hering et al., 2014; Irwin et al., 1985; Kong et al., 1993; Kwan et al., 1991; Lewis et al., 2012; LuValle et al., 1992; Mendler et al., 1989; Morrison et al., 1996; Muller-Glauser et al., 1986; Oshima et al., 1989; Poole et al., 1984; Ricard-Blum et al., 1982; Schmid and Linsenmayer, 1985; Shen, 2005; Shoham et al., 2016; Treilleux et al., 1992; Vornehm et al., 1996; Wilusz et al., 2012) but only a few studies (Shoham et al., 2016; Wilusz et al., 2012) have analysed some of these collagen structures using the improved resolution offered by confocal microscopy. The main novelty of the present study is that it revealed, for the first time, to the authors' knowledge, the emergence and maturation of key cartilage and bone collagens, in high resolution, at key locations across the entire rudiment, including the joint regions, at three of the most developmentally significant stages of skeletogenesis.

Collagens I, II, III, V, VI, X and XI in the developing mouse humerus demonstrated dynamic spatial distribution and structural changes between the three stages studied, namely TS22, TS25 and TS27, as summarised graphically in Fig. 12. At TS22, collagen I was strongly present at the proximal end of the rudiment and in the perichondrium, while by TS25 and TS27 immunopositivity was mostly confined to the mineralised regions. Collagen $\mathrm{V}$ also spatially rearranged from the diaphyseal region at TS22 to the mineralised regions at TS25 and TS27, with changes in the structural organisation from pericellular localisation at TS22 to a fibrillar organisation at TS27. Collagen II was primarily present in the non-mineralised cartilage. There were striking differences in collagen II structure and organisation between regions and between stages, with highly organised fibrillar network of collagen II matrix present in the TS27 humeral head and condyles when compared to TS22 and TS25. The structural organisations of collagens III and VI changed from dense perinuclear localisation at TS22 to a distinctive pericellular localisation with cristaelike arrangements within individual chondrons by TS25. Strong immunopositivity for collagen $X$ was detected in the mid-diaphysis of the TS22 rudiments with a hexagonal lattice-like framework. By TS25 and TS27, collagen $X$ was restricted to the growth plate, still with a distinctive lattice-like pattern. At TS22, collagen XI formed a fibrillar network throughout the entire rudiment but this structure was only retained in the humeral head and the condyles at TS25 and TS27. In the growth plates at TS25 and TS27, collagen XI fibres were highly organised within the transverse and longitudinal septa. Collagens I, III, VI, IX and XI were localised to the tendon attachment sites throughout development. The future articular cartilage region was demarcated by pronounced collagens II and VI expression at TS27.

The only collagen that was relatively homogenous over the stages studied was collagen IX, which was consistently expressed close to the cells throughout the rudiment. The lack of collagen IX expression throughout the ECM was unexpected given that collagen IX copolymerises with collagen II and XI to form a heteropolymer. Several previous studies using collagen IX antibodies (and similar HA digestion protocols) have also reported immunolocalisation to the pericellular matrix of chondrocytes in epiphyseal cartilage of developing or adult skeletal tissues (Duance et al., 1982; Evans et al., 1983; Hartmann et al., 1983; Poole et al., 1984; Ricard-Blum et al., 1982). In contrast, one study did report extensive collagen IX staining throughout the cartilage matrix in the chick tibiotarsus (Irwin et al., 1985). Therefore, it is possible that the pericellular staining observed in the present and other studies could be due to staining 

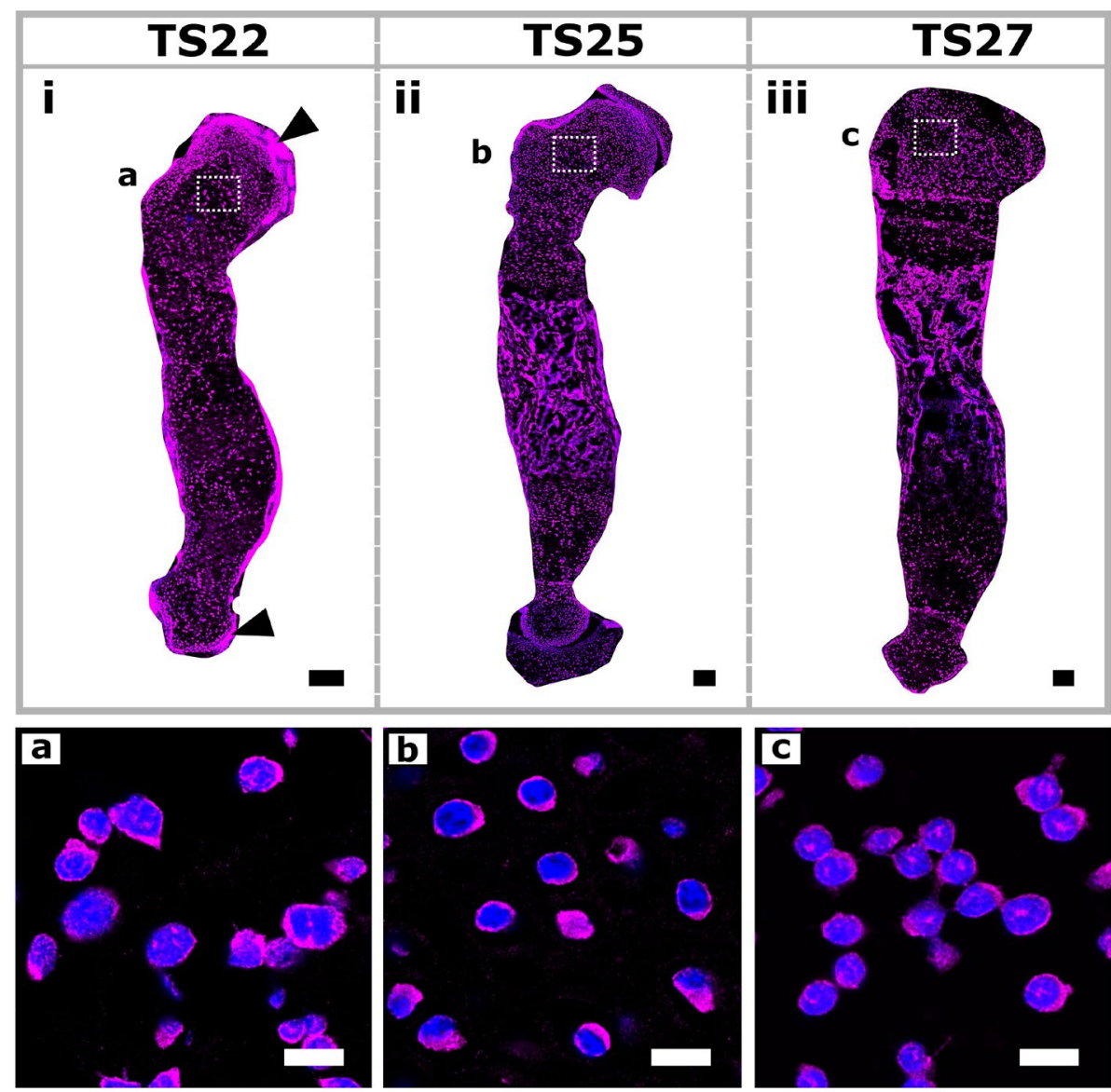

DAPI Collagen IX
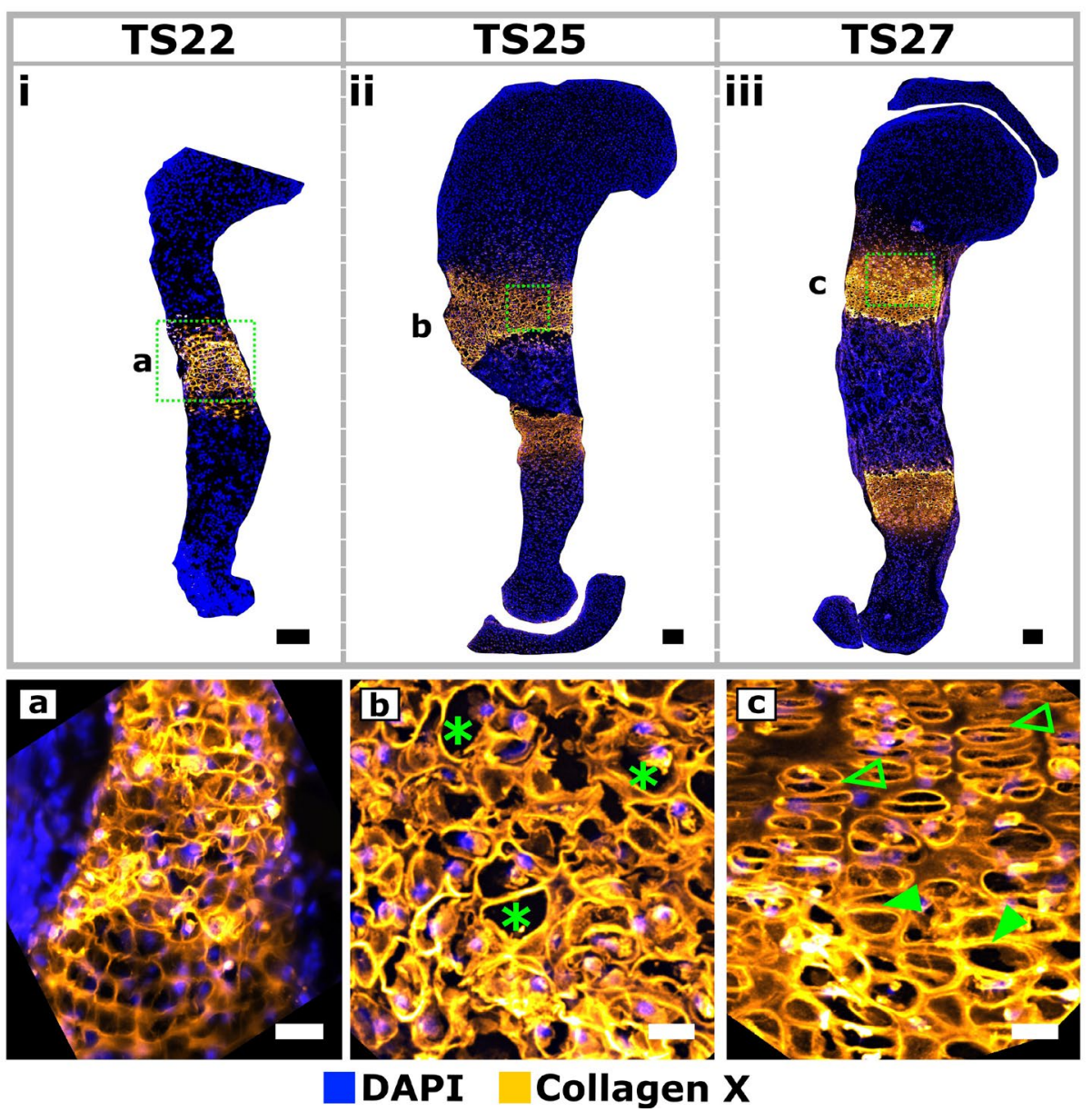

Fig. 7. Perinuclear expression of collagen IX did not exhibit pronounced dynamic changes in tissue distribution and structure during the developmental stages studied. (i) Filled black arrows indicate the strong collagen IX immunopositivity in the proximal and distal ends of the rudiment at TS22. White dotted boxes represent the specific areas where the higher magnification images were taken. Scale bars: $100 \mu \mathrm{m}$ for i-iii and $10 \mu \mathrm{m}$ for a-c.
Fig. 8. Collagen $X$ was localised in a membrane-like configuration around chondrocytes with increasing complexity in structure through development. Green dotted boxes represent the specific areas where the higher magnification images were taken. (b) Asterisks: capsular localisation of collagen X. (c) Hollow arrows: columnar arrangement in the pre-hypertrophic region of the growth plate; filled arrows: convex arrangement in the hypertrophic region of the growth plate. Scale bars: $100 \mu \mathrm{m}$ for i-iii and $25 \mu \mathrm{m}$ for $\mathbf{a}-\mathbf{c}$. 

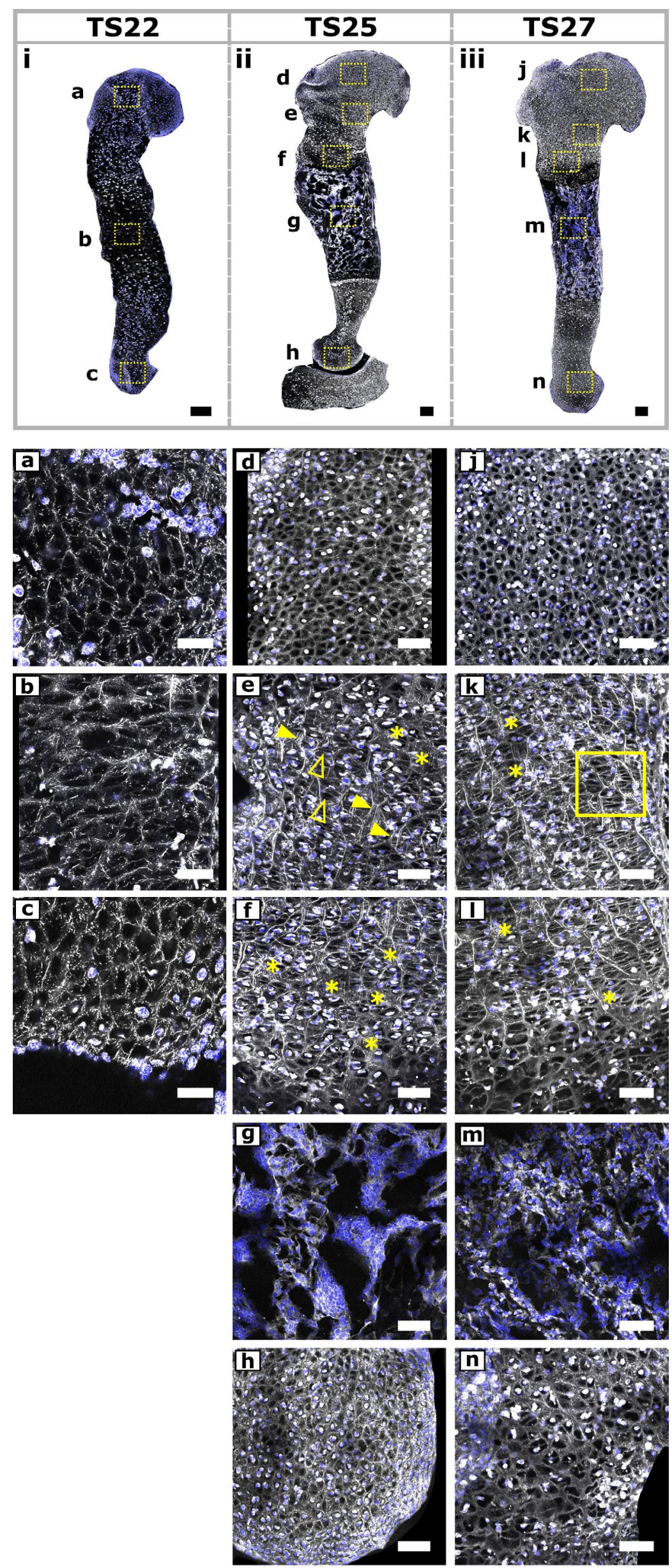

\section{DAPI Collagen XI}

Fig. 9. Collagen XI immunopositivity was detected in the non-mineralised cartilage at all stages with dramatic changes in the structural organisation between regions and stages. Collagen XI matrix had an interlacing conformation at (a-c) TS22 which persisted until $(\mathbf{d}, \mathbf{j})$ TS27 in the humeral head and $(\mathbf{h}, \mathbf{n})$ humeral condyles. In the TS25 and TS27 growth plates, collagen XI fibres were arranged in the longitudinal and transverse septa. Yellow dotted boxes represent the specific areas where the higher magnification images were taken. (e) Filled arrows: collagen XI fibres in the longitudinal septa were oriented parallel to each other with a small inter-fibre space between them; hollow arrows: collagen XI in the transverse septa. $(\mathbf{e}, \mathbf{f}, \mathbf{k}, \mathbf{l})$ Asterisks: characteristic short fibres. (k) Square: collagen XI matrix organisations in the transverse and longitudinal septa of the TS27 proliferative growth plate region. Scale bars: $100 \mu \mathrm{m}$ for $\mathbf{i}-\mathbf{i i i}$ and $50 \mu \mathrm{m}$ for a-n. 
of only a fraction of the collagen IX structures which the antibody could penetrate and have access to. In addition, a proportion of the antigenic sites on individual molecules might be concealed by the collagen molecule orientation within a fibril and non-collagenous proteins could mask the collagen IX antigenic sites. Finally, given that collagen IX covalently binds the surface of collagen II molecules (Wu et al., 2010), analysing collagen interactions by attenuated total reflection-Fourier transform infrared (ATR-FTIR) spectroscopy might have resulted in a more complex pattern for collagens IX and II.

A key feature of the dynamic spatial rearrangement of the collagens was the segregation of the staining for collagen $X$ from collagens II and IX with the progression of growth plate maturation. The progression of chondrocytes through hypertrophy involved strong collagen $\mathrm{X}$ expression and reduced collagens II and IX expression near the advancing marrow cavity region of the growth plate. Consistent with this, Irwin et al. (1985) found that chondrocyte hypertrophy involves both mRNA acquisition for collagen $X$ and extensive diminution of collagen IX mRNA and protein expression. Two other prior studies have shown that collagen II distribution diminishes in the hypertrophic cartilage of juvenile chickens (Oshima et al., 1989) and in cultured chondrocytes (Castagnola et al., 1988). Given that collagen II is a suppressor of chondrocyte hypertrophy (Lian et al., 2019), such a reduction at the growth plate is logical. Presence of collagen $X$ at the growth plate region was as expected given that collagen $X$ assembly plays an important role in the modification of cartilage matrix for subsequent bone formation by endochondral ossification (Kwan et al., 1991). There is evidence for the importance of collagen $X$ in matrix-vesicle-mediated calcification of hypertrophic cartilage and its contribution to the establishment of the haematopoietic niche at the chondro-osseous junction (Sweeney et al., 2010). Collagen $X$ results
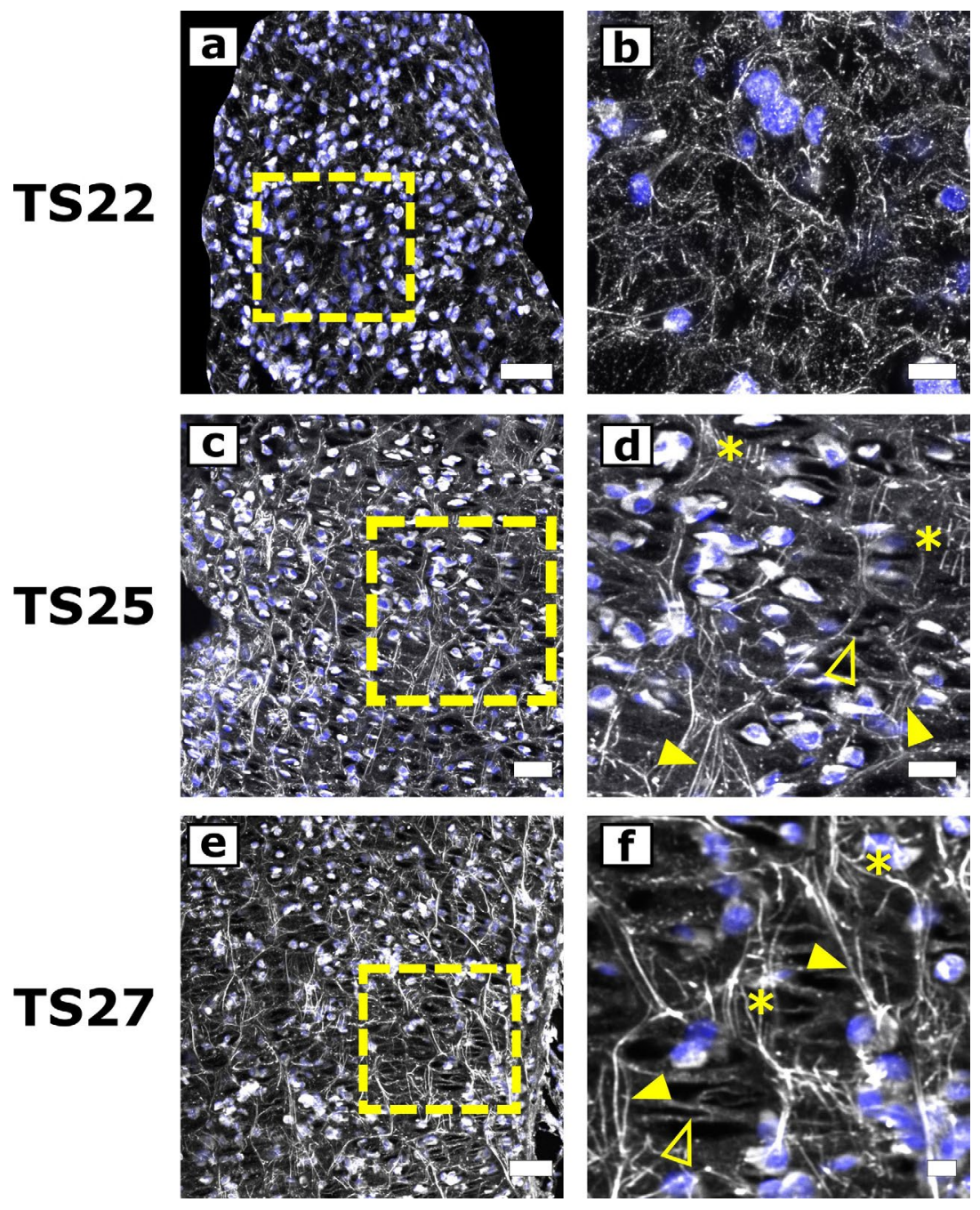

Fig. 10. Distinctive collagen XI fibre organisation in the growth plates of the embryonic humerus during limb development. Yellow dotted boxes (left panels) represent the specific areas where the higher magnification images (right panels) swere taken. At TS22, collagen XI formed a fibrillar network. However, by TS25 and TS27, the initial interlacing arrangement and size of the fibrils became progressively more complex. Filled arrowheads: collagen XI fibres in the longitudinal septa were oriented parallel to each other with a small inter-fibre space between them; hollow arrowheads: collagen XI in the transverse septa; asterisks: short collagen XI fibres. 


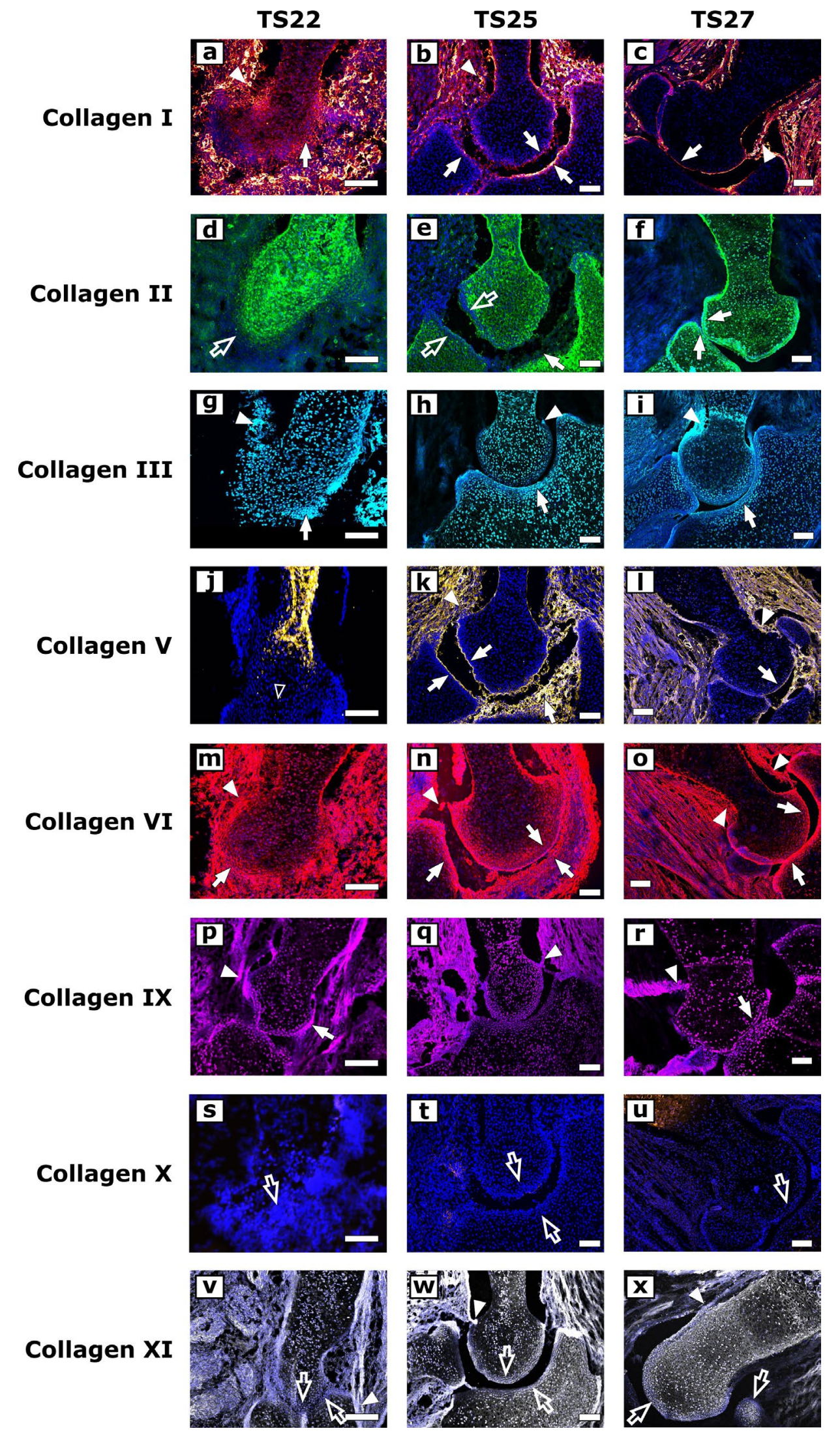

Fig. 11. Collagen distributions at the developing humero-ulnar joint of the mouse forelimb. Collagens I, III, VI, IX and XI were localised to the tendon attachment sites (arrow heads) throughout development. Collagens I and V were localised to the perichondrium but not within the humeral condyles. At TS27, collagens II, III, VI and XI were expressed both at the future articular cartilage region and in the epiphysis. Collagen $X$ was not expressed either in the humeral condyles or at the future articular cartilage regions at any of the stages studied. Solid arrow heads: collagen localisation at the tendon-bone attachment sites. Solid arrows: collagen localisation in the future articular cartilage regions. Hollow arrows: lack of collagen localisation in the future articular cartilage regions. Scale bars: $100 \mu \mathrm{m}$. 
corroborate a previous study in mice showing initiation of collagen $X$ gene expression at E13.5 and expression in the hypertrophic chondrocytes at E16.5 (Kong et al., 1993). However, a novel finding from the present study was the expression of collagen $X$ protein in the pre-hypertrophic region of the growth plate at TS27.

A second key observation was the segregation of the stainings for collagens I and V from collagens II and XI with the progression of ossification. Collagens I and V were strongly expressed in the mid-diaphysis of the TS22 rudiments preceding the onset of mineralisation, while only little staining, if any, was observed in this region for collagens II and XI. As mineralisation progressed, collagens I and $\mathrm{V}$ were localised exclusively in the mineralised cartilage while collagens II and XI were not retained in the mineralised regions. Localisations of all these collagens corroborate previous findings and are in agreement with the roles of these collagens in the mineralisation process. The results on collagen I immunolocalisation in the bone collar were also in agreement with previous reports (Shoham et al., 2016). Collagen I is necessary for guiding the organisation and growth of hydroxyapatite crystals during mineralisation, and mutations in collagen I severely impair formation, organisation and orientation of apatite crystals, leading to a significant increase in bone brittleness (Fratzl et al., 1996). Collagen V is important for controlling collagen I fibril diameter in bone (Glimcher et al., 1980; Niyibizi and Eyre, 1989; Wenstrup et al., 2004). Finally, an in vitro study showed that higher levels of collagen II correlate with reduced mineralisation capacity (Jubeck et al., 2009), which may explain why collagen II is not retained in the mineralised cartilage.

Another key observation was the change in collagen structural organisation and complexity with

\section{Cartilage}
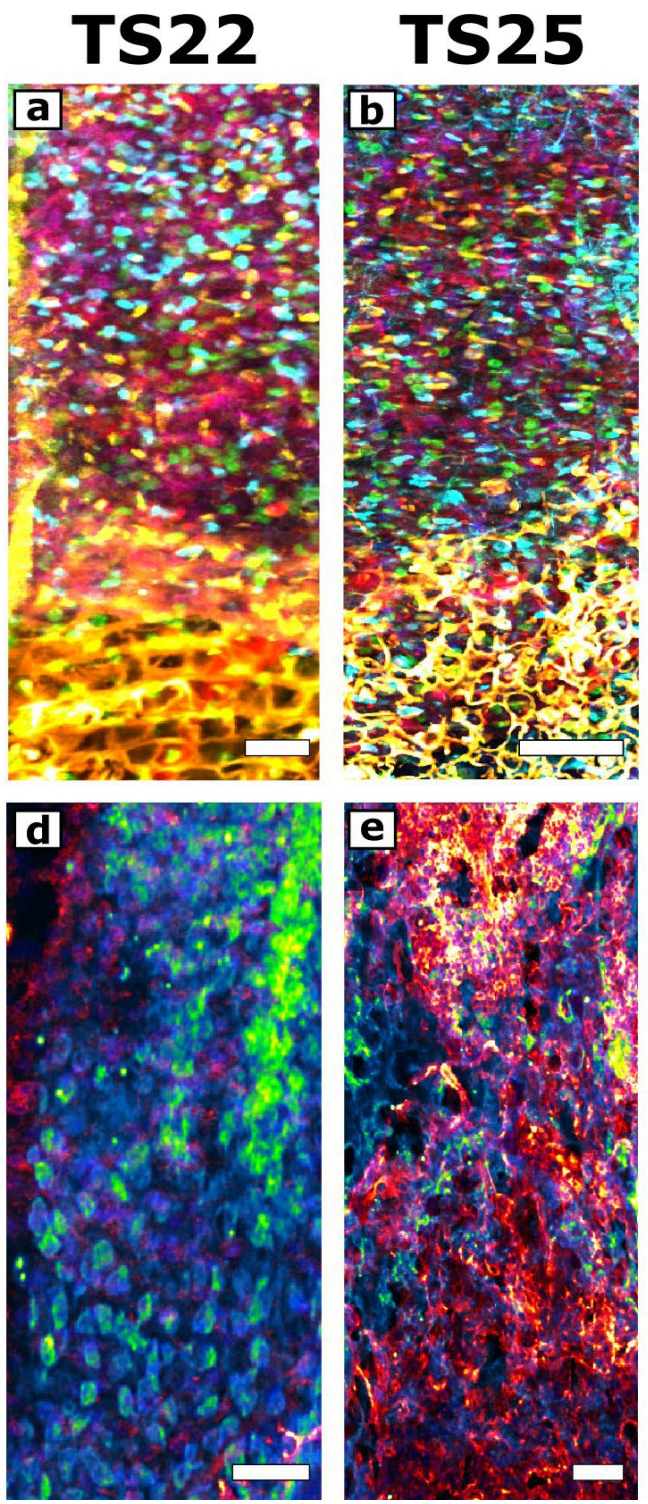
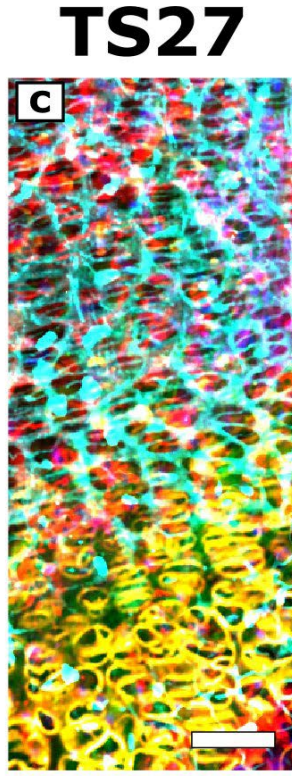

DAPI

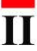

II

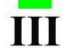

VI

IX

$\mathbf{X}$

XI

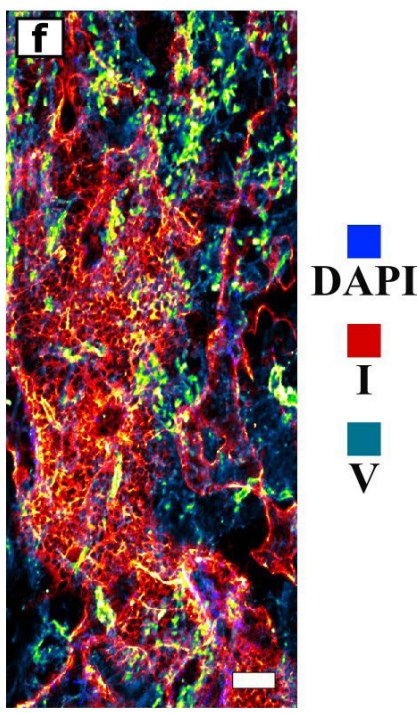

Fig. 12. Composite images showing the overall changes in localisation patterns of collagens predominantly found in the cartilaginous and mineralising regions across three developmental stages. (a-c) Cartilage; TS22: mid-diaphysis, TS25 and TS27: growth plate. (d-f) Mineralising region. Prominent cartilage collagens: collagens II (red), III (green), VI (magenta), IX (yellow), X (orange) and XI (cyan). Prominent collagens in the mineralising regions: collagens I (red) and V (blue-green). Scale bars: $50 \mu \mathrm{m}$. 
maturation. At TS22, no obvious arrangement for collagen II was observed, whereas by TS27, collagen II formed a fibrillar network at both epiphyses and proximo-distal-oriented bundle in the growth plate. Similarly, no obvious demarcation was observed between a pericellular and extracellular expression of collagen VI at TS22. By TS25, distinctive chondrons encapsulating single chondrocytes were observed at all regions of the rudiment. At all stages, collagen $X$ had a membrane-like configuration, although this configuration differed between the stages. At TS22, collagen $X$ had a hexagonal lattice-like appearance, which changed to a more capsular configuration surrounding hypertrophic chondrocytes at TS25 and TS27. At TS27, collagen $X$ had a columnar arrangement in the pre-hypertrophic region and a convex arrangement in the hypertrophic region. However, the most radical changes were for collagen XI fibres at the growth plate regions. The formation of the collagen XI framework preceded the collagen II framework establishment and was in agreement with previous findings according to which collagen II polymerises on a template of collagen XI (Wu et al., 2010). At TS22, collagen XI fibres were organised in an interlacing network throughout the rudiment that persisted until TS27 in the humeral heads and condyles. However, at TS25 and TS27, in the growth plate, collagen XI fibres had three distinguishing organisations: (1) fibres in the transverse septa, (2) parallel-oriented fibres in the longitudinal septa with small inter-fibrillar space between the fibres, (3) highly oriented shorter fibres organised in parallel within the longitudinal septa. With maturation (going from TS25 to TS27), these fibre types became more pronounced especially along the longitudinal septa, with preferential orientation of these dense bundles along the proximodistal axis.

The study was not without its limitations. Since all analyses were performed on or prior to TS27, the spatiotemporal distribution of the key collagens after TS27 was not within the remit of the study. However, it would have been interesting to continue the investigation postnatally and characterise the collagen distribution patterns up until maturation of the long bone. Furthermore, the study focused on describing the structural organisation and tissue distribution patterns of the key collagens, but other aspects of collagen organisation, such as collagen cross-linking or interactions between collagens II, IX and XI, likely also change with tissue maturation. Perhaps analysis of the cross-linking or collagen interactions would have provided a better understanding of the collagen IX structure in the wider ECM. $12 \mu$ m-thick frozen sections were used to characterise the collagen organisations and, therefore, the 3D organisation of the different collagens was not characterised. Future studies will combine tissue clearing techniques for immunofluorescence with light sheet microscopy in order to characterise the collagen distributions through greater depths of the rudiment. The distribution of collagen IIB isoform was investigated, which was prominently localised throughout the cartilage matrix from TS22. Previous studies have proposed that collagen IIA is the main isoform produced at E12.5, with IIB being reported as being barely detectable (Hering et al., 2014; Lewis et al., 2012; McAlinden, 2014) at that stage. By E16.5, the IIA isoform is mainly localised to the resting zone of the cartilage whereas IIB is present throughout the cartilage including the growth plates. Collagen IIB results corroborated these findings, but it would have been interesting to compare the IIB localisation patterns with those of IIA. Finally, the changes in the relative amount of each collagen type across development or changes in the collagen bundle orientations were not quantified. Nonetheless, while recognising the limitations of the analysis, the present study addressed a key gap in knowledge describing the dynamic changes in collagen structural organisations across the various regions of the rudiment during skeletogenesis.

A major setback to the attempts to repair and regenerate the injured cartilage is the limited knowledge of the ECM architecture and mechanical properties; the incorporation of developmentally inspired constituents and properties will help to promote robust cartilage and bone regeneration. Indeed, collagen XI was very recently shown to be a potent positive regulator of chondrogenesis in vitro (Li et al., 2018). The present study provided a comprehensive characterisation of the tissue distribution and structural organisation of the key cartilage and bone collagens in various regions of the rudiment and across the key stages of prenatal skeletal development and included the lesser studied collagens III, V, IX and XI. The high-resolution localisation of the collagens presented enhances the understanding of the emergence and establishment of the ECM and of the contribution of individual matrix components to skeletal development, with important implications for understanding diseases and regeneration of skeletal tissues.

\section{Acknowledgements}

We thank the Imperial College London FILM Facility, Stephen M Rothery, Andreas Bruckbauer and David Gaboriau for their support with microscopy. This research was funded by the European Research Council under the European Union's Seventh Framework Programme (ERC Grant agreement number 336306). The Facility for Imaging by Light Microscopy (FILM) at Imperial College London is part-supported by funding from the Wellcome Trust (grant 104931/Z/14/Z) and BBSRC (grant BB/ L015129/1). The funders had no role in study design, data collection and analysis, decision to publish or preparation of the manuscript. We also appreciate the reviewers' constructive comments which helped us to improve the quality of the manuscript. 


\section{References}

Alexopoulos LG, Youn I, Bonaldo P, Guilak F (2009) Developmental and osteoarthritic changes in Col6a1-knockout mice: biomechanics of type VI collagen in the cartilage pericellular matrix. Arthritis Rheum 60: 771-779.

Alford AI, Kozloff KM, Hankenson KD (2015) Extracellular matrix networks in bone remodeling. Int J Biochem Cell Biol 65: 20-31.

Archer CW, Morrison H, Pitsillides AA (1994) Cellular aspects of the development of diarthrodial joints and articular cartilage. J Anat 184 (Pt 3): 447-456.

Aszodi A, Chan D, Hunziker E, Bateman JF, Fassler R (1998) Collagen II is essential for the removal of the notochord and the formation of intervertebral discs. J Cell Biol 143: 1399-1412.

Beighton P, De Paepe A, Steinmann B, Tsipouras P, Wenstrup RJ (1998) Ehlers-Danlos syndromes: revised nosology, Villefranche, 1997. Ehlers-Danlos National Foundation (USA) and Ehlers-Danlos Support Group (UK). Am J Med Genet 77: 31-37.

Billinghurst RC, Buxton EM, Edwards MG, McGraw MS, McIlwraith CW (2001) Use of an antineoepitope antibody for identification of type-II collagen degradation in equine articular cartilage. Am J Vet Res 62: 1031-1039.

Birk DE (2001) Type V collagen: heterotypic type $\mathrm{I} / \mathrm{V}$ collagen interactions in the regulation of fibril assembly. Micron 32: 223-237.

Blaschke UK, Eikenberry EF, Hulmes DJ, Galla HJ, Bruckner P (2000) Collagen XI nucleates self-assembly and limits lateral growth of cartilage fibrils. J Biol Chem 275: 10370-10378.

Blumbach K, Bastiaansen-Jenniskens YM, DeGroot J, Paulsson M, van Osch GJ, Zaucke F (2009) Combined role of type IX collagen and cartilage oligomeric matrix protein in cartilage matrix assembly: cartilage oligomeric matrix protein counteracts type IX collagen-induced limitation of cartilage collagen fibril growth in mouse chondrocyte cultures. Arthritis Rheum 60: 3676-3685.

Castagnola P, Dozin B, Moro G, Cancedda R (1988) Changes in the expression of collagen genes show two stages in chondrocyte differentiation in vitro. J Cell Biol 106: 461-467.

Christensen SE, Coles JM, Zelenski NA, Furman BD, Leddy HA, Zauscher S, Bonaldo P, Guilak F (2012) Altered trabecular bone structure and delayed cartilage degeneration in the knees of collagen VI null mice. PLoS One 7: e33397. DOI: 10.1371/journal. pone.0033397.

Clearfield D, Nguyen A, Wei M (2018) Biomimetic multidirectional scaffolds for zonal osteochondral tissue engineering via a lyophilization bonding approach. J Biomed Mater Res A 106: 948-958.

Duance VC, Shimokomaki M, Bailey AJ (1982) Immunofluorescence localization of type-M collagen in articular cartilage. Bioscience Reports 2: 223-227.

Eltawil NM, Ahmed S, Chan LH, Simpson A, Hall AC (2018) Chondroprotection in models of cartilage injury by raising the temperature and osmolarity of irrigation solutions. Cartilage 9: 313-320.

Evans HB, Ayad S, Abedin MZ, Hopkins S, Morgan K, Walton KW, Weiss JB, Holt PJL (1983) Localization of collagen types and fibronectin in cartilage by immunofluorescence. Ann Rheum Dis 42: 575-581.

Eyre D (2002) Collagen of articular cartilage. Arthritis Res 4: 30-35.

Foolen J, van Donkelaar C, Nowlan N, Murphy P, Huiskes R, Ito K (2008) Collagen orientation in periosteum and perichondrium is aligned with preferential directions of tissue growth. J Orthop Res 26: 1263-1268.

Forlino A, Cabral WA, Barnes AM, Marini JC (2011) New perspectives on osteogenesis imperfecta. Nat Rev Endocrinol 7: 540-557.

Fratzl P, Paris O, Klaushofer K, Landis WJ (1996) Bone mineralization in an osteogenesis imperfecta mouse model studied by small-angle $X$-ray scattering. J Clin Invest 97: 396-402.

Garofalo S, Vuorio E, Metsaranta M, Rosati R, Toman D, Vaughan J, Lozano G, Mayne R, Ellard J, Horton W, et al. (1991) Reduced amounts of cartilage collagen fibrils and growth plate anomalies in transgenic mice harboring a glycine-to-cysteine mutation in the mouse type II procollagen alpha 1-chain gene. Proc Natl Acad Sci U S A 88: 9648-9652.

Gelse K, Poschl E, Aigner T (2003) Collagens structure, function, and biosynthesis. Adv Drug Deliv Rev 55: 1531-1546.

Gentili C, Cancedda R (2009) Cartilage and bone extracellular matrix. Curr Pharm Des 15: 1334-1348.

Glimcher MJ, Shapiro F, Ellis RD, Eyre DR (1980) Changes in tissue morphology and collagen composition during the repair of cortical bone in the adult chicken. J Bone Joint Surg Am 62: 964-973.

Hartmann DJ, Magliore H, Ricard-Blum S, Joffre A, Couble M-L, Ville G, Herbage D (1983) Light and electron immunoperoxidase localization of minor disulfidebonded collagens in fetal calf epiphyseal cartilage. Coll Relat Res 3: 349-357.

Helminen HJ, Säämänen AM, Salminen $H$, Hyttinen MM (2002) Transgenic mouse models for studying the role of cartilage macromolecules in osteoarthritis. Rheumatology 41: 848-856.

Hering TM, Wirthlin L, Ravindran S, McAlinden A (2014) Changes in type II procollagen isoform expression during chondrogenesis by disruption of an alternative 5' splice site within Col2a1 exon 2. Matrix Biol 36: 51-63.

Holyoak DT, Otero M, Armar NS, Ziemian SN, Otto A, Cullinane D, Wright TM, Goldring SR, Goldring MB, van der Meulen MCH (2018) Collagen XI mutation lowers susceptibility to load-induced cartilage damage in mice. J Orthop Res 36: 711-720.

Hosseininia S, Weis MA, Rai J, Kim L, Funk S, Dahlberg LE, Eyre DR (2016) Evidence for enhanced collagen type III deposition focally in the territorial matrix of osteoarthritic hip articular cartilage. Osteoarthritis Cartilage 24: 1029-1035. 
Irwin MH, Silvers SH, Mayne R (1985) Monoclonal antibody against chicken type IX collagen: preparation, characterization, and recognition of the intact form of type IX collagen secreted by chondrocytes. J Cell Biol 101: 814-823.

Jubeck B, Muth E, Gohr CM, Rosenthal AK (2009) Type II collagen levels correlate with mineralization by articular cartilage vesicles. Arthritis Rheum 60: 2741-2746.

Kadler KE, Hill A, Canty-Laird EG (2008) Collagen fibrillogenesis: fibronectin, integrins, and minor collagens as organizers and nucleators. Curr Opin Cell Biol 20: 495-501.

Kamekura S, Hoshi K, Shimoaka T, Chung U, Chikuda H, Yamada T, Uchida M, Ogata N, Seichi A, Nakamura K, Kawaguchi H (2005) Osteoarthritis development in novel experimental mouse models induced by knee joint instability. Osteoarthritis Cartilage 13: 632-641.

Kong RY, Kwan KM, Lau ET, Thomas JT, BootHandford RP, Grant ME, Cheah KS (1993) Intronexon structure, alternative use of promoter and expression of the mouse collagen $\mathrm{X}$ gene, Col10a-1. Eur J Biochem 213: 99-111.

Kwan AP, Cummings CE, Chapman JA, Grant ME (1991) Macromolecular organization of chicken type X collagen in vitro. J Cell Biol 114: 597-604.

Levingstone TJ, Thompson E, Matsiko A, Schepens A, Gleeson JP, O'Brien FJ (2016) Multi-layered collagen-based scaffolds for osteochondral defect repair in rabbits. Acta Biomater 32: 149-160.

Lewis R, Ravindran S, Wirthlin L, Traeger G, Fernandes RJ, McAlinden A (2012) Disruption of the developmentally-regulated Col2a1 pre-mRNA alternative splicing switch in a transgenic knock-in mouse model. Matrix Biol 31: 214-226.

Li A, Wei Y, Hung C, Vunjak-Novakovic G (2018) Chondrogenic properties of collagen type $\mathrm{XI}$, a component of cartilage extracellular matrix. Biomaterials 173: 47-57.

Li SW, Prockop DJ, Helminen H, Fassler R, Lapvetelainen T, Kiraly K, Peltarri A, Arokoski J, Lui H, Arita M, JKhillan JS (1995a) Transgenic mice with targeted inactivation of the Col2 alpha 1 gene for collagen II develop a skeleton with membranous and periosteal bone but no endochondral bone. Genes Dev 9: 2821-2830.

Li Y, Lacerda DA, Warman ML, Beier DR, Yoshioka $\mathrm{H}$, Ninomiya Y, Oxford JT, Morris NP, Andrikopoulos K, Ramirez F, Wardell BB, Lifferth GD, Teuscher EC, Woodward SR, Taylor BA, Seegmiller RE, Olsen BR (1995) A fibrillar collagen gene, Col11a1, is essential for skeletal morphogenesis. Cell 80: 423-430.

Lian C, Wang X, Qiu X, Wu Z, Gao B, Liu L, Liang G, Zhou H, Yang X, Peng Y, Liang A, Xu C, Huang D, Su P (2019) Collagen type II suppresses articular chondrocyte hypertrophy and osteoarthritis progression by promoting integrin beta1-SMAD1 interaction. Bone Res 7: 8. DOI: 10.1038/s41413-0190046-y.
Longley R, Ferreira AM, Gentile P (2018) Recent approaches to the manufacturing of biomimetic multiphasic scaffolds for osteochondral regeneration. Int J Mol Sci 19. DOI: 10.3390/ijms19061755.

LuValle P, Daniels K, Hay ED, Olsen BR (1992) Type $X$ collagen is transcriptionally activated and specifically localized during sternal cartilage maturation. Matrix 12: 404-413.

McAlinden A (2014) Alternative splicing of type II procollagen: IIB or not IIB? Connect Tissue Res 55: 165-176.

Mendler M, Eich-Bender SG, Vaughan L, Winterhalter KH, Bruckner P (1989) Cartilage contains mixed fibrils of collagen types II, IX, and XI. J Cell Biol 108: 191-197.

Morrison EH, Ferguson MW, Bayliss MT, Archer CW (1996) The development of articular cartilage: I. The spatial and temporal patterns of collagen types. J Anat 189 (Pt 1): 9-22.

Müller-Glauser W, Humbel B, Glatt M, Sträuli P, Winterhalter KH, Bruckner P (1986) On the role of type IX collagen in the extracellular matrix of cartilage: type IX collagen is localized to intersections of collagen fibrils. J Cell Biol 102: 1931-1939.

Niyibizi C, Eyre DR (1989) Bone type V collagen: chain composition and location of a trypsin cleavage site. Connect Tissue Res 20: 247-250.

Olsen BR (1995) Mutations in collagen genes resulting in metaphyseal and epiphyseal dysplasias. Bone 17: 45s-49s.

Oshima O, Leboy PS, McDonald SA, Tuan RS, Shapiro IM (1989) Developmental expression of genes in chick growth cartilage detected by in situ hybridization. Calcif Tissue Int 45: 182-192.

Paletzki R, Gerfen CR (2015) Whole mouse brain image reconstruction from serial coronal sections using FIJI (ImageJ). Curr Protoc Neurosci 73: 1.25.2121. DOI: $10.1002 / 0471142301 . n s 0125 s 73$.

Parsons P, Gilbert SJ, Vaughan-Thomas A, Sorrell DA, Notman R, Bishop M, Hayes AJ, Mason DJ, Duance VC (2011) Type IX collagen interacts with fibronectin providing an important molecular bridge in articular cartilage. J Biol Chem 286: 34986-34997.

Poole CA, Flint MH, Beaumont BW (1984) Morphological and functional interrelationships of articular cartilage matrices. J Anat 138 (Pt 1): 113-138.

Ricard-Blum S, Hartmann DJ, Herbage D, PayenMeyran C, Ville G (1982) Biochemical properties and immunolocalization of minor collagens in foetal calf cartilage. FEBS Lett 146: 343-347.

Rozario T, DeSimone DW (2010) The extracellular matrix in development and morphogenesis: a dynamic view. Dev Biol 341: 126-140.

Schmid TM, Linsenmayer TF (1985) Immunohistochemical localization of short chain cartilage collagen (type $\mathrm{X}$ ) in avian tissues. J Cell Biol 100: 598-605.

Shen G (2005) The role of type X collagen in facilitating and regulating endochondral ossification of articular cartilage. Orthod Craniofac Res 8: 11-17. 
Shoham AB, Rot C, Stern T, Krief S, Akiva A, Dadosh T, Sabany H, Lu Y, Kadler KE, Zelzer E (2016) Deposition of collagen type I onto skeletal endothelium reveals a new role for blood vessels in regulating bone morphology. Development 143: 3933-3943.

Stoop R, van der Kraan PM, Buma P, Hollander AP, Poole AR, van den Berg WB (1999) Denaturation of type II collagen in articular cartilage in experimental murine arthritis. Evidence for collagen degradation in both reversible and irreversible cartilage damage. J Pathol 188: 329-337.

Sweeney E, Roberts D, Corbo T, Jacenko O (2010) Congenic mice confirm that collagen $X$ is required for proper hematopoietic development. PLoS One 5: e9518. DOI: 10.1371/journal.pone.0009518.

Treilleux I, Mallein-Gerin F, le Guellec D, Herbage D (1992) Localization of the expression of type I, II, III collagen, and aggrecan core protein genes in developing human articular cartilage. Matrix 12: 221-232.

von Der Mark K (2006) Chapter 1 - Structure, biosynthesis and gene regulation of collagens in cartilage and bone. In: Dynamics of bone and cartilage metabolism. 2nd edition. Editors: Seibel MJ, Robins SP, Bilezikian JP. Academic Press, Burlington. pp: 3-40.

Vandenberg P, Khillan JS, Prockop DJ, Helminen H, Kontusaari S, Ala-Kokko L (1991) Expression of a partially deleted gene of human type II procollagen (COL2A1) in transgenic mice produces a chondrodysplasia. Proc Natl Acad Sci U S A 88: 7640-7644.

Vornehm SI, Dudhia J, von der Mark K, Aigner T (1996) Expression of collagen types IX and XI and other major cartilage matrix components by human fetal chondrocytes in vivo. Matrix Biology 15: 91-98.

Walker GD, Fischer M, Gannon J, Thompson RC Jr, Oegema TR Jr (1995) Expression of type-X collagen in osteoarthritis. J Orthop Res 13: 4-12.

Wenstrup RJ, Florer JB, Brunskill EW, Bell SM, Chervoneva I, Birk DE (2004) Type V collagen controls the initiation of collagen fibril assembly. J Biol Chem 279: 53331-53337.

Wilusz RE, DeFrate LE, Guilak F (2012) Immunofluorescence-guided atomic force microscopy to measure the micromechanical properties of the pericellular matrix of porcine articular cartilage. J R Soc Interface 9: 2997-3007.

Wu J-J, Weis MA, Kim LS, Eyre DR (2010) Type III collagen, a fibril network modifier in articular cartilage. J Biol Chem 285: 18537-18544.

Yuan S, Chan HCS, Filipek S, Vogel H (2016) PyMOL and inkscape bridge the data and the data visualization. Structure 24: 2041-2042.

\section{Discussion with Reviewers}

Audrey McAlinden: You stated that your findings have important implications for understanding skeletal disease and skeletal regeneration. Can you elaborate on this?

Authors: Recent advances in biomaterial design, such as 3D bio-printing, enable exquisite control over biomaterial composition and structure. One way in which the current study and its follow-on work could be leveraged is to incorporate collagens and other ECM constituents into developmentally inspired scaffolds, which may lead to a more effective healing and regeneration of cartilage or bone through recapitulation of developmental processes. Furthermore, a better understanding of the normal sequence of collagen establishment will also potentially lead to new insights into conditions such as OA and osteoporosis. There are certain mouse lines such as those with Col2a1 mutations that are known to be at higher risk of osteoporosis or OA; elucidating collagen development in such lines will reveal if such diseases could have a developmental origin and open avenues for novel treatments.

Audrey McAlinden: You commented that you have analysed three of the most developmentally significant stages of skeletogenesis. Why exactly are these stages in particular so important?

Authors: TS22 (E13.5) is the latest stage at which the rudiment is fully cartilaginous, while TS27 (E18.5) is the latest prenatal stage that can be reliably analysed. Characterising TS25 (E16.5) enabled the study of growth plate progression and early-stage mineralisation.

Audrey McAlinden: What experimental approach/ project would you pursue next to follow on the findings presented in the present manuscript?

Authors: Key future research directions include investigation of the 3D organisation of collagens over prenatal development, study of the non-collagenous ECM, including GAGs and proteoglycans, characterisation of the mechanical properties of developing cartilage (and their relationship with ECM composition) and determination of the role of mechanical forces in the emergence of the ECM.

Farshid Guilak: While the collagens are clearly important during development, have the authors examined other important matrix proteins and/or proteoglycans?

Authors: Non-collagenous proteins, such as proteoglycans, play a vital role in the function of adult cartilage and are likely to exhibit dynamic changes during development. We are excited to investigate the development of non-collagenous proteins (particularly proteoglycans) in future studies.

Editor's note: The Scientific Editor responsible for this paper was Martin Stoddart. 